\title{
Hepatic CEACAM1 expression indicates donor liver quality and prevents early transplantation injury
}

\author{
Kojiro Nakamura, ${ }^{1}$ Shoichi Kageyama, ${ }^{1}$ Fady M. Kaldas, ${ }^{1}$ Hirofumi Hirao, ${ }^{1}$ Takahiro Ito, ${ }^{1}$ Kentaro Kadono, ${ }^{1}$ Kenneth J. Dery, ${ }^{1}$ \\ Hidenobu Kojima,' David W. Gjertson, ${ }^{2,3}$ Rebecca A. Sosa, ${ }^{3}$ Maciej Kujawski, ${ }^{4}$ Ronald W. Busuttil, ${ }^{1}$ Elaine F. Reed, ${ }^{3}$ \\ and Jerzy W. Kupiec-Weglinski'
}

'Department of Surgery, Division of Liver and Pancreas Transplantation, Dumont-UCLA Liver Transplant Center, ${ }^{2}$ Department of Biostatistics, UCLA School of Public Health, ${ }^{3}$ Department of Pathology and Laboratory Medicine, David Geffen School of Medicine at UCLA, Los Angeles, California, USA. ${ }^{4}$ Department of Molecular Immunology, Beckman Research Institute of City of Hope, Duarte, California, USA.

\begin{abstract}
Although CEACAM1 (CC1) glycoprotein resides at the interface of immune liver injury and metabolic homeostasis, its role in orthotopic liver transplantation (OLT) remains elusive. We aimed to determine whether/how CEACAM1 signaling may affect hepatic ischemia-reperfusion injury (IRI) and OLT outcomes. In the mouse, donor liver CC1 null mutation augmented IRI-OLT $($ CC1-KO $\rightarrow$ WT) by enhancing ROS expression and HMCB1 translocation during cold storage, data supported by in vitro studies where hepatic flush from CC1-deficient livers enhanced macrophage activation in bone marrow-derived macrophage cultures. Although hepatic CC1 deficiency augmented cold stress-triggered ASK1/p-p38 upregulation, adjunctive ASK1 inhibition alleviated IRI and improved OLT survival by suppressing p-p38 upregulation, ROS induction, and HMGB1 translocation $($ CC1-KO $\rightarrow$ WT), whereas ASK1 silencing (siRNA) promoted cytoprotection in cold-stressed and damage-prone CC1-deficient hepatocyte cultures. Consistent with mouse data, CEACAM1 expression in 60 human donor liver biopsies correlated negatively with activation of the ASK1/p-p38 axis, whereas low CC1 levels associated with increased ROS and HMCB1 translocation, enhanced innate and adaptive immune responses, and inferior early OLT function. Notably, reduced donor liver CEACAM1 expression was identified as one of the independent predictors for early allograft dysfunction (EAD) in human OLT patients. Thus, as a checkpoint regulator of IR stress and sterile inflammation, CEACAM1 may be considered as a denominator of donor hepatic tissue quality, and a target for therapeutic modulation in OLT recipients.
\end{abstract}

\section{Introduction}

Orthotopic liver transplantation (OLT) has become the standard of care for patients with end-stage liver disease and hepatic malignancies. However, early allograft dysfunction (EAD), an important cause of morbidity and mortality for liver transplant recipients, is not uncommon, with the incidence at approximately $25 \%$, varying between centers from $10.8 \%$ to $36.3 \%$ (1). Ischemia-reperfusion injury (IRI), a leading cause of EAD, represents a major risk factor for acute and chronic rejection, and a contributing factor in severe organ shortage available for transplantation. These adverse effects become even more substantial in expanded-criteria liver transplants, including those from marginal and deceased donors, and donors without a heartbeat. However, despite obvious clinical importance, the mechanisms that account for liver IRI are not well appreciated, and novel strategies to improve clinical outcomes and expand the donor pool to alleviate organ shortage are warranted (2).

\section{Related Commentary: p. 2192}

Authorship note: KN, SK, FMK, and HH are co-first authors. Conflict of interest: The authors have declared that no conflict of interest exists. Copyright: ( 2020 , American Society for Clinical Investigation. Submitted: September 3, 2019; Accepted: January 30, 2020; Published: April 20, 2020. Reference information: J Clin Invest. 2020;130(5):2689-2704. https://doi.org/10.1172/JCl133142.
In the pathophysiology of liver IRI, primary hepatic insult during ex vivo cold storage triggers stressed cells to secrete danger-associated molecular patterns (DAMPs), such as histone $\mathrm{H} 3$ and high-mobility group box 1 (HMGB1). The latter mount a cascade of innate immune-dominated inflammatory responses during the reperfusion phase, accelerating the hepatocellular OLT damage and undermining long-term survival and clinical outcomes. Although cell death during the reperfusion phase appears to proceed primarily via oncotic necrosis $(3,4)$, consistent with our own and others' studies demonstrating the contribution of apoptosis and necroptosis in IRI pathology (5-7), the hepatocellular death program at the reperfusion phase may encompass a mixture of diverse molecular mechanisms. Although the restoration of hepatic blood flow is the principal cause of tissue injury, pretransplant cold storage itself can trigger organ damage as well (8). Apoptosis signal-regulating kinase 1 (ASK1), a member of the MAP3K family, becomes activated by a wide range of extra- and intracellular stressors. Activation of p38 MAPK was reported in cold-induced tissue injury $(9,10)$, whereas a recent study has identified tissuespecific contribution of ASK1 and p-p38 pathways in cell death signaling (11). The role of the ASK1/p-p38 axis in the mechanism of liver transplant damage has not been studied before.

The carcinoembryonic antigen-related cell adhesion molecule 1 (CEACAM1, CC1; CD66a) is a transmembrane glycoprotein expressed on epithelial, endothelial, and immune cells. Original- 
ly identified as a substrate of the insulin receptor tyrosine kinase in rat hepatocytes $(12,13)$, and abundantly expressed by the bile capillary side of hepatocytes in rodents and humans, CEACAM1 is involved in hepatocyte differentiation/liver regeneration, and increasingly recognized as the master regulator of insulin clearance, while preventing metabolic disorders, such as diabetes and nonalcoholic steatohepatitis (NASH) $(14,15)$. The emerging evidence of hepatic CEACAM1 residing at the central hub of immune liver injury and metabolic homeostasis (16) provides the rationale to study its relevance in IRI-OLT in the current study.

Here, we asked as to whether and how hepatic CEACAM1 may affect susceptibility of liver grafts to peritransplant stress and thus influence clinical outcomes in mouse and human OLT recipients. Our findings document the functional role of CEACAM1 signaling to alleviate graft damage during cold preservation, mitigate posttransplant IRI, and improve early clinical outcomes, while identifying an underlying ASK1-p38 molecular mechanism of cytoprotection. In the experimental arm, we employed a clinically relevant mouse OLT model with extended ex vivo cold storage, to show that liver cell-specific CEACAM1 signaling protected OLT against cold stress, alleviated IRI, and improved overall survival. By demonstrating that adjunctive ASK1 inhibition prevented otherwise fulminant hepatocellular damage due to hepatic CC1 genetic ablation, we have identified stress-activated ASK1 as a key CEACAM1 downstream molecule in liver graft protection. In the clinical arm of 60 human liver transplant patients, cold-stored human donor livers with decreased CEACAM1 levels exhibited increased ASK1 signaling and inferior post-OLT function. Notably, reduced hepatic CEACAM1 expression was identified as one of the independent predictors for EAD in human OLT recipients. Thus, as a checkpoint regulator of IR-stress and hepatic sterile inflammation, CEACAM1 may serve not only as a target for therapeutic OLT modulation, but also as a denominator of donor liver tissue quality. The latter may have a major clinical impact on OLT outcomes, as currently there is no reliable way to preoperatively assess donor organ quality.

\section{Results}

Hepatic CC1 null mutation exacerbates IRI in mouse OLT. We first aimed to determine the influence of graft-specific disruption of CEACAM1 signaling on the severity of hepatic IRI in a clinically relevant mouse OLT model with extended ex vivo cold storage $\left(4^{\circ} \mathrm{C} / 18\right.$ hours), which mimics the marginal human liver graft scenario. At 6 hours after transplantation into WT recipients, CC1deficient $\left(\mathrm{CC}^{-/} ; \mathrm{KO}\right)$ liver grafts $(n=6)$ exhibited increased sinusoidal congestion, edema vacuolization, and hepatocellular necrosis (Figure 1A); enhanced Suzuki's histological IRI grading (WT $\rightarrow$ $\mathrm{WT}=3.5 \pm 1.0$ vs. $\mathrm{CC} 1-\mathrm{KO} \rightarrow \mathrm{WT}=6.0 \pm 1.3, P=0.0005$, Figure $1 \mathrm{~B})$; higher serum levels of alanine aminotransferase (sALT) and aspartate aminotransferase (sAST) (sAST: WT $\rightarrow \mathrm{WT}=3053 \pm 501$ vs. $\mathrm{CC} 1-\mathrm{KO} \rightarrow \mathrm{WT}=6097 \pm 1324 \mathrm{IU} / \mathrm{L}, P<0.0001$; sALT: $\mathrm{WT} \rightarrow$ $\mathrm{WT}=6616 \pm 1065$ vs. $\mathrm{CC} 1-\mathrm{KO} \rightarrow \mathrm{WT}=9807 \pm 2655, P=0.0087$; Figure 1C); and elevated frequency of TUNEL-positive necrotic/ apoptotic cells $(\mathrm{WT} \rightarrow \mathrm{WT}=46.6 \pm 4.9$ vs. $\mathrm{CC} 1-\mathrm{KO} \rightarrow \mathrm{WT}=83.7$ $\pm 14.7 / \mathrm{HPF}, P<0.0001$; Figure $1, \mathrm{D}$ and $\mathrm{E}$ ) as compared with CC1 proficient (WT $\rightarrow \mathrm{WT}$ ) grafts $(n=6)$. Thus, disruption of CEACAM1 signaling in the donor liver augmented IRI and enhanced hepatocellular death in murine OLT.
Hepatic CC1 ablation enhances IR-inflammatory phenotype in mouse OLT. Since the release of DAMPs, such as HMGB1, from damaged cells triggers a cascade of inflammatory cytokine/ chemokine events, which further aggravate organ damage (17), we aimed to evaluate the impact of graft $C C 1$ deficiency on the release of HMGB1 and accompanied innate-immune response in our model. At 6 hours after reperfusion, CC1-KO liver grafts $(\mathrm{CC1}-\mathrm{KO} \rightarrow \mathrm{WT}$ ) showed higher serum HMGB1 levels (Figure 1F) and increased frequency of intragraft infiltration by CD11b-positive (macrophage)/Ly6G-positive (neutrophil) cells (Figure 1, D and $\mathrm{G}$ ), along with elevated serum MCP1 (Figure $1 \mathrm{~F}$ ) and hepatic mRNA levels coding for MCP1, CXCL1, CXCL2, and CXCL10 (Figure $1 \mathrm{H}$ ), as compared with controls $(\mathrm{WT} \rightarrow \mathrm{WT}$ ). These data indicate the importance of graft CC1 signaling to suppress secretion of DAMPs, mitigate innate immune activation, and alleviate hepatocellular damage in IR-stressed OLT.

Hepatic CC1 deletion augments cell damage by enhancing reactive oxygen species (ROS) and HMGB1 translocation during liver cold storage. Although restoration of blood flow at reperfusion is the principal cause of liver IRI (17), cold storage itself can also trigger hepatocellular damage (8). Having demonstrated the importance of graft $C C 1$ expression on HMGB1 release in OLT (Figure 1F), we next asked whether CEACAM1 may affect graft injury and HMGB1 signaling during ex vivo cold storage (before revascularization). Herein, we focused on the liver effluent obtained by flushing the liver with physiological saline $(2 \mathrm{~mL})$ via a cuff placed at portal vein immediately after 18 hours of cold stimulation (Figure 2A). Indeed, the flush from CC1-deficient livers contained increased HMGB1 and histone H3 levels as compared with CC1-proficient (WT) livers (Figure 2B), suggesting higher susceptibility of CC1-KO grafts to peritransplant cold stress. Since the generation of ROS is one of the principal factors leading to cell death in cold-stored tissue (8, 18), we then evaluated 4-Hydroxynonenal (4HNE, a ROS metabolite) levels in our model. Cold stimulation alone increased $4 \mathrm{HNE}$ expression, mainly in hepatic parenchymal cells, whereas CC1 deficiency markedly enhanced 4HNE upregulation (Figure 2C, upper panel, red stains). Moreover, cold stress led to cytoplasmic HMGB1 translocation, while CC1 disruption further augmented extranuclear HMGB1 localization (Figure 2C, middle panel). Thus, disruption of hepatic CC1 signaling increased effluence of damage-associated molecules (HMGB1/histone H3) while enhancing 4HNE expression and extranuclear HMGB1 translocation during cold storage. These data suggest that graft-specific CEACAM1 was essential to inhibit local ROS generation and prevent cell damage in the early hepatic cold storage phase. Noteworthy, cold stimulation alone did not affect the frequency of TUNEL-positive cells in WT or CC1-KO liver grafts (Figure 2C, lower panel), implying hepatic damage leading to DAMPs release (Figure 2B) was likely distinct from apoptotic/necrotic cell death.

Hepatic flush from CC1-KO cold-stored mouse livers enhances macrophage activation in vitro. Since the leakage of DAMPs from a cold-stored graft is not only a tissue damage indicator, but may also trigger innate inflammatory response, we next asked whether and how liver flush may affect bone marrow-derived macrophage (BMDM) cultures (Figure 2, A and D). Indeed, liver flush from cold-stored WT livers was found to increase mRNA levels coding for MCP1, CXCL2 and CXCL10, whereas liver flush from CC1- 
A
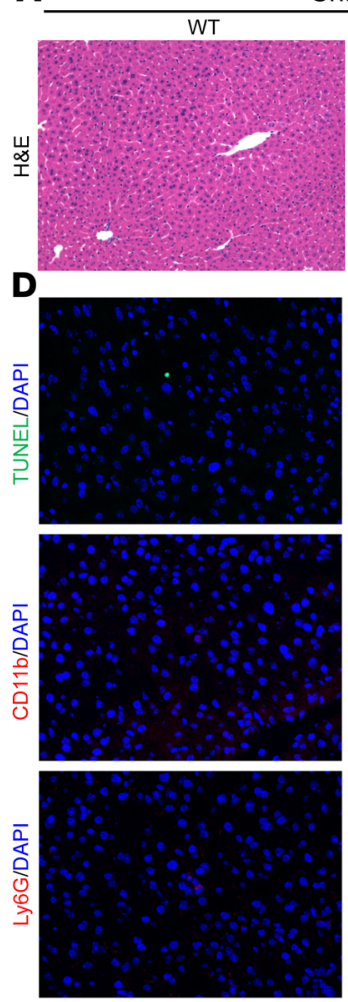

$\Delta$ WT: sham

$\boldsymbol{\nabla}$ CC1-KO: sham

O OLT: WT $\rightarrow$ WT

- OLT: CC1-KO $\rightarrow$ WT

E

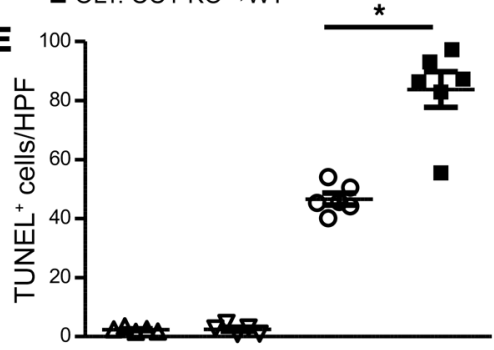

Sham
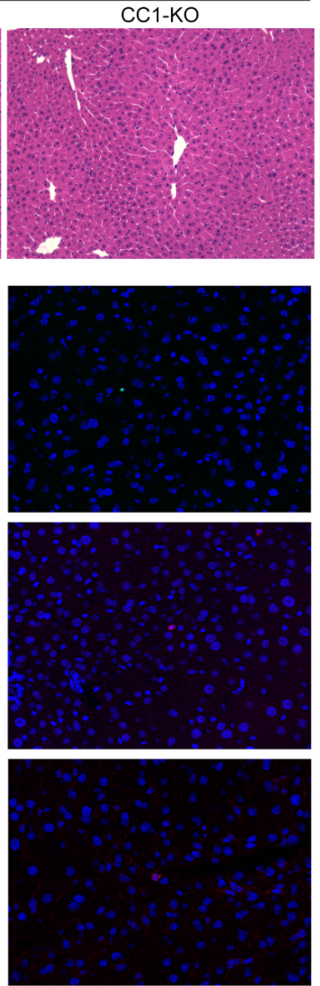

OLT
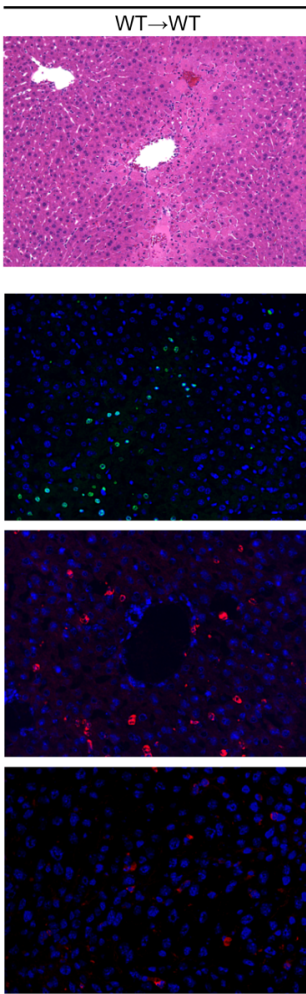

$\mathrm{CC} 1-\mathrm{KO} \rightarrow \mathrm{WT}$
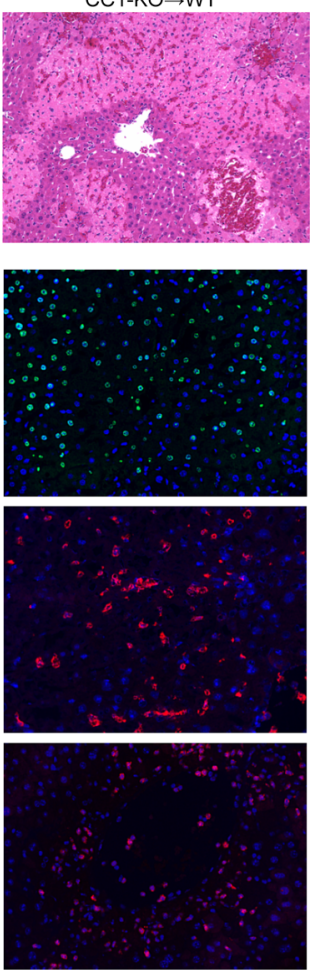

B
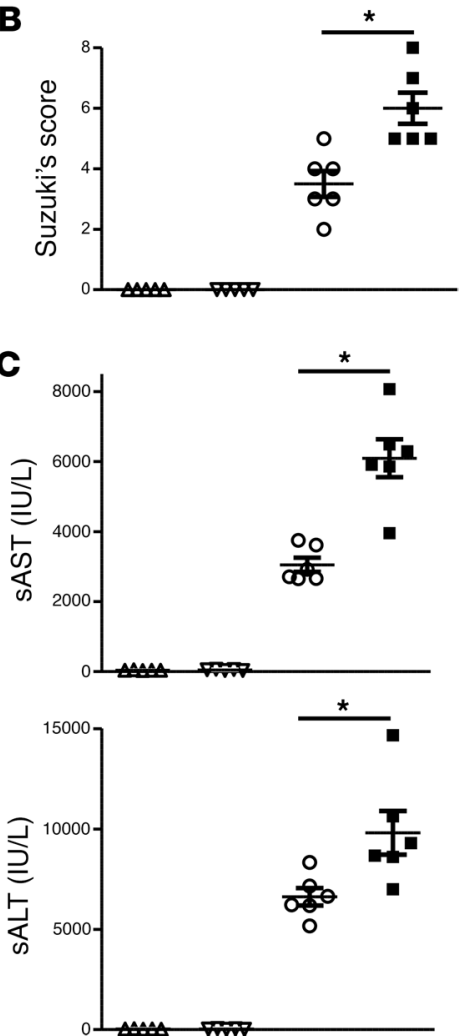

G

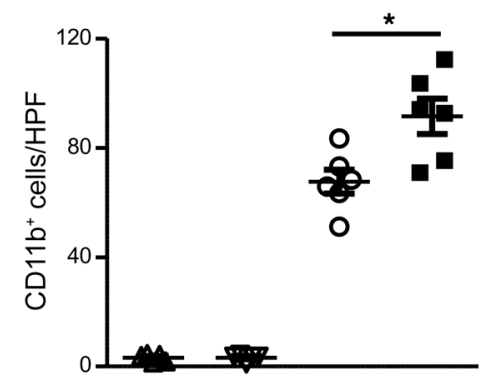

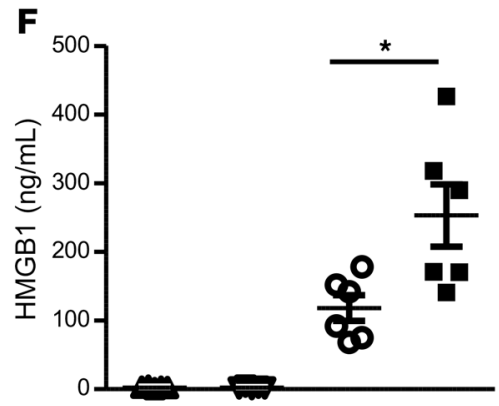
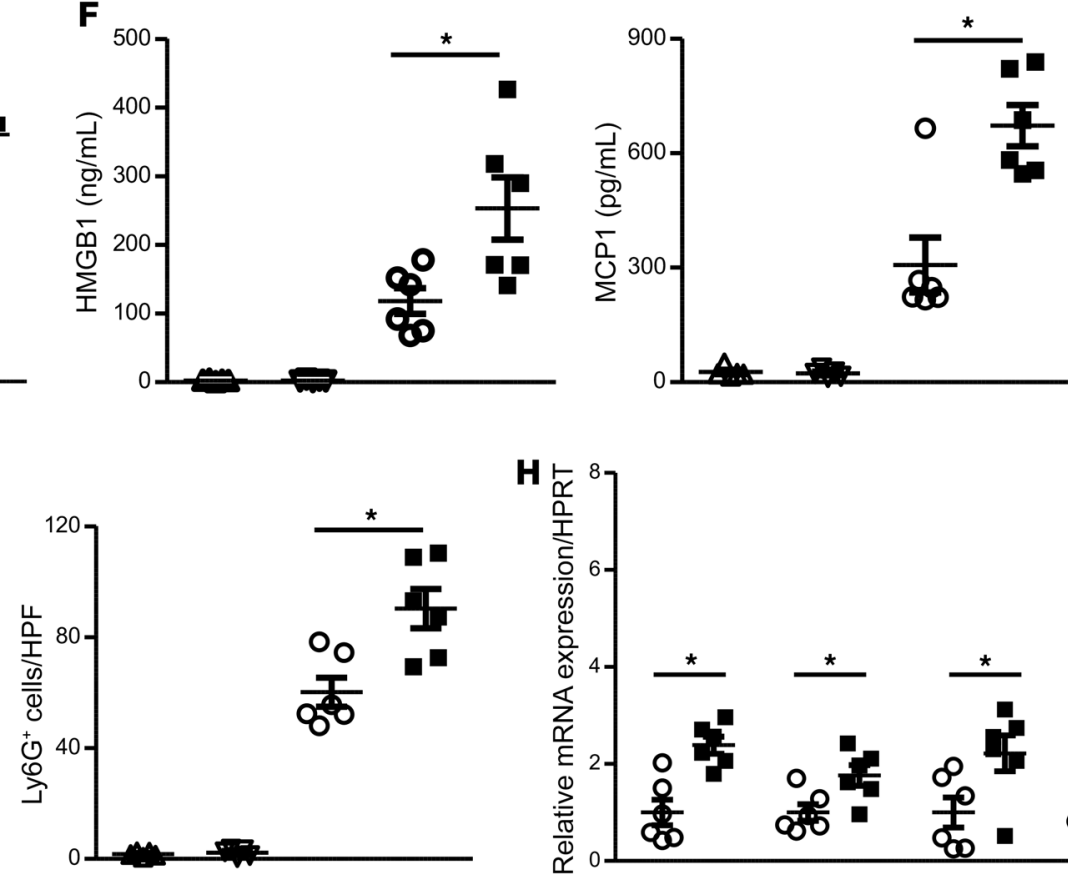

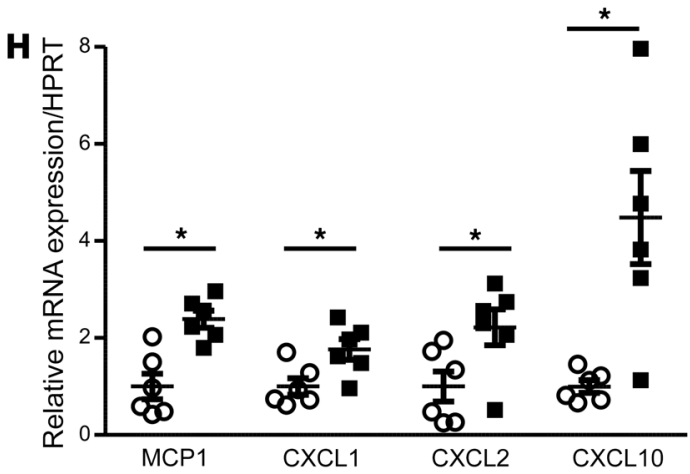

Figure 1. Hepatic CC1-null mutation exacerbates hepatocellular damage and inflammatory response in IR-stressed mouse OLT. Livers from groups of WT and CC1-KO C57BL/6 donor mice, stored in UW solution ( $4^{\circ} \mathrm{C} / 18$ hours), were transplanted to WT C57BL/6 recipient mice. OLT and serum samples were analyzed 6 hours after reperfusion. The sham group underwent the same procedures except for OLT. (A) Representative H\&E staining (original magnification $\times 100$ ). (B) Suzuki's histological grading of liver IRI. (C) SAST and SALT levels (IU/L). (D) Representative TUNEL staining and immunohistochemical staining of OLT-infiltrating CD11 b+ and Ly6G+ cells (original magnification $\times 200)$. (E) Quantification of TUNEL-positive cells/HPF. (F) Serum HMCB1 (ng/mL) and MCP1 $(\mathrm{pg} / \mathrm{mL}$ ) levels measured by ELISA. (C) Quantification of hepatic CD11b+ and Ly6G+ cells/HPF. (H) Real-time reverse transcription PCR-assisted (qRT-PCR-assisted) detection of mRNA coding for MCP1, CXCL1, CXCL2, and CXCL10 in OLT. Data were normalized to HPRT gene expression. Data are mean $\pm \mathrm{SD} .{ }^{*} P<0.05$, 1-way ANOVA followed by Tukey's HSD test (B, C, and E-C) or Student's $t$ test $(\mathbf{H}), n=5$-6/group. 


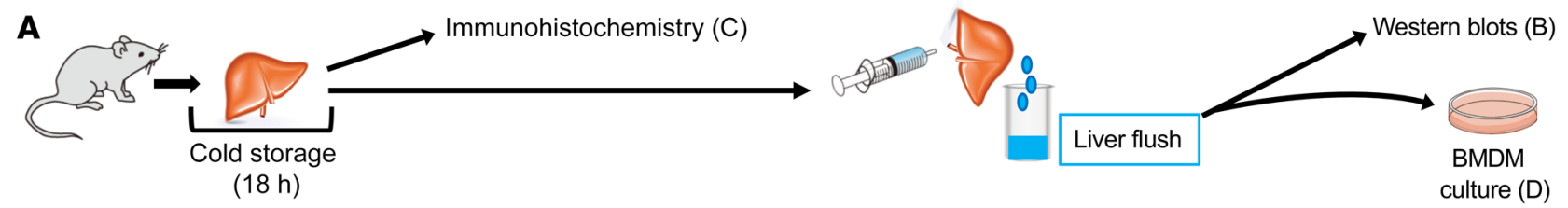

B
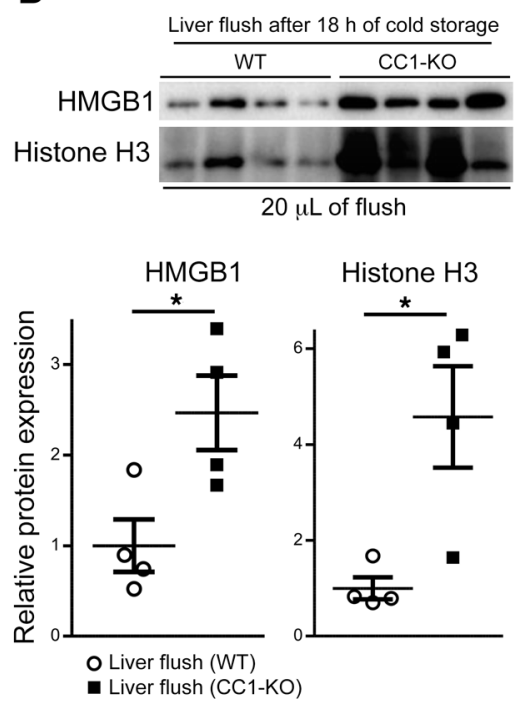

C
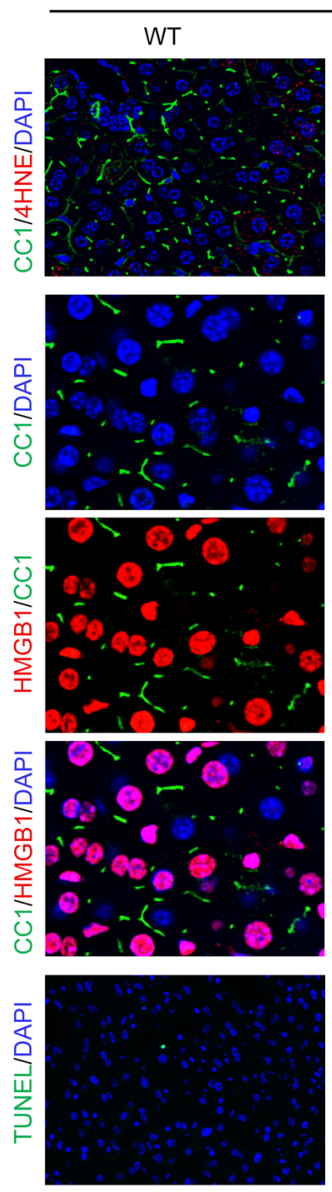

Cold-stored $(18 \mathrm{~h})$ liver
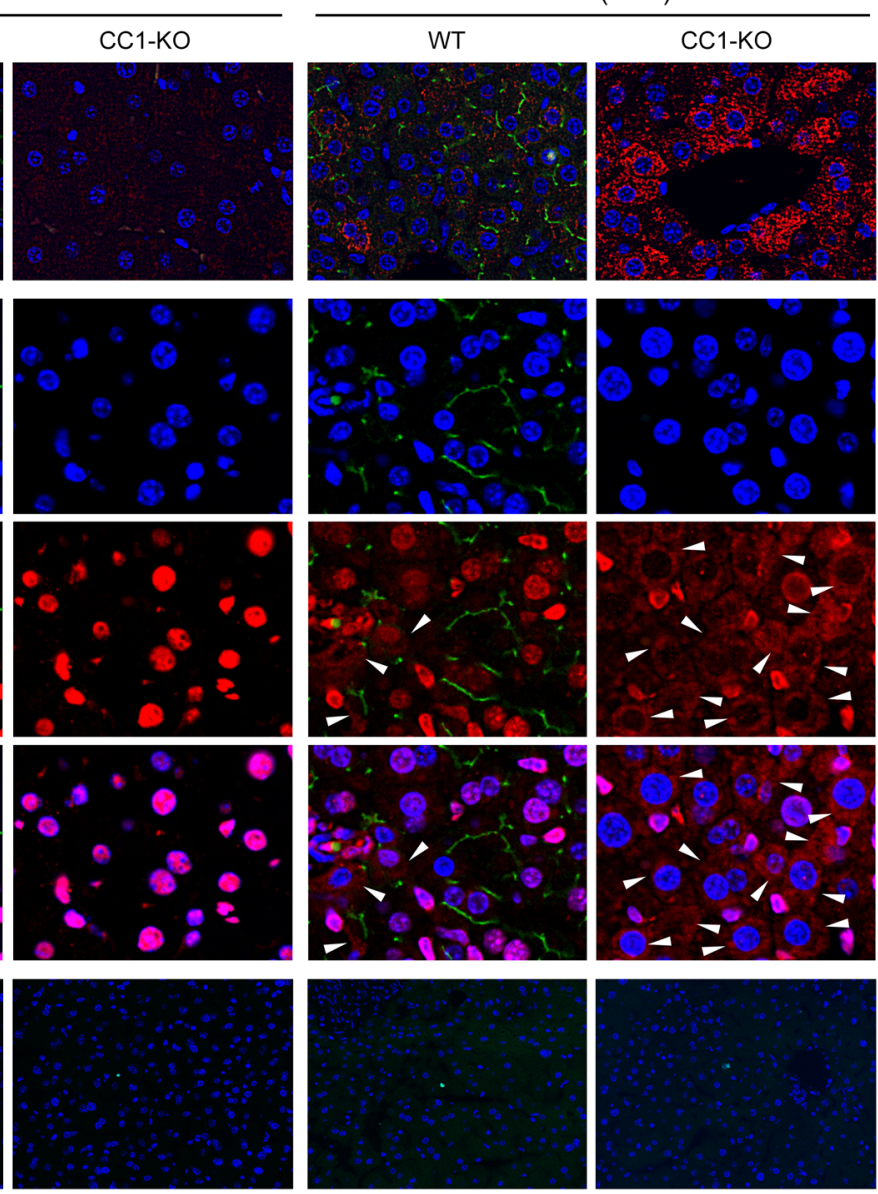

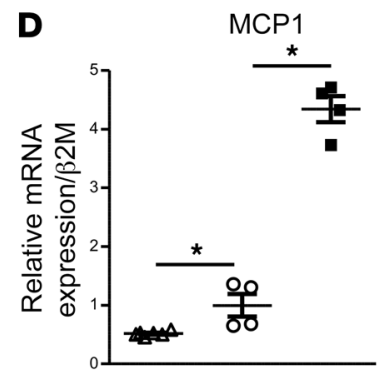

CXCL2

CXCL10
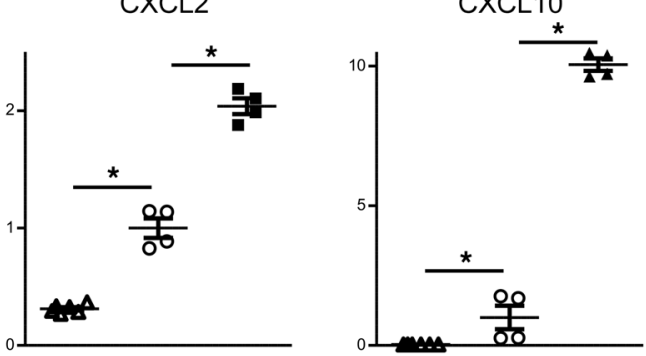

$\triangle \mathrm{BMDMs}$

- BMDMs + liver flush (WT)

- BMDMs + liver flush (CC1-KO)

BMDM culture \pm stimulation with cold-stored liver flush

Figure 2. CC1 ablation in cold-stored livers enhances ROS and HMGB1 translocation/release to liver flush, which further increases inflammatory gene program in macrophage cultures. (A) Groups of WT and CC1-KO liver grafts stored in UW solution $\left(4^{\circ} \mathrm{C} / 18\right.$ hours) were perfused with physiological saline $(2 \mathrm{~mL}$ ) via a cuff placed at the portal vein to collect liver flush from inferior vena cava. (B) Liver flush samples (20 $\mu$ L) from cold-stressed WT or CC1deficient livers were screened by Western blots for HMGB1/Histone H3 levels ( $n=4$ /group, ${ }^{*} P<0.05$, Student's $t$ test). (C) WT or CC1-KO liver grafts were collected after cold storage $\left(4^{\circ} \mathrm{C} / 18\right.$ hours). Representative $(n=3 /$ group) immunohistochemical staining of CC1/4HNE (a ROS metabolite), CC1/HMCB1, and TUNEL is shown. Arrowheads indicate extranuclear HMGB1 localization. (D) BMDM cultures (WT) were stimulated (6 hours) with liver flush obtained from WT or CC1 KO cold-stored grafts. QRT-PCR-assisted detection of mRNA coding for MCP1, CXCL2, CXCL10 with $\beta 2 \mathrm{M}$ normalization ( $n=4-6$, ${ }^{*} P<0.05,1$-way ANOVA followed by Tukey's HSD test). 
KO livers further enhanced the proinflammatory gene expression program as compared with WT cultures. Thus, in the absence of hepatic CC1 signaling, cold stimulation triggered a more pronounced inflammatory response by hepatic flush in vitro, as compared with CC1-proficient (WT) liver flush.

Hepatic CC1 null mutation augments cold stress-triggered upregulation of the ASK1/p-p38 signaling axis. To seek a molecular signaling pathway that underlies cold-related hepatic damage, we contrasted murine WT livers at resting state (naive), after cold storage $\left(4^{\circ} \mathrm{C} / 18\right.$ hours), and after OLT reperfusion (Figure $3 \mathrm{~A}$ ). Cleavage of caspase-3, an essential step to execute apoptosis, was pronounced at 3 hours after reperfusion, while cold stress alone failed to increase cleaved caspase-3 expression. Consistently, TUNEL-positive (apoptotic/necrotic) cells were increased in OLT (Figure 1D) but not during cold storage alone (Figure 2C). In addition, cold stimulation did not affect RIP3 (a marker of necroptosis) expression (Figure 3B). These findings indicate that the molecular mechanism of cold stress-triggered liver graft damage was likely to be distinct from apoptosis, necrosis, or necroptosis. Since recent reports have demonstrated tissue-specific importance of ASK1/ p-p38 signaling in cold-stress cell damage $(10,11)$, we focused on the ASK1/p-p38 axis in our model. Interestingly, the markedly increased hepatic ASK1 and p-p38 expression seen after cold storage $\left(4^{\circ} \mathrm{C} / 18\right.$ hours) in WT livers decreased in OLT at 3 hours after reperfusion (Figure $3 \mathrm{C}$ ). In marked contrast, in the absence of $\mathrm{CC1}$ signaling, liver grafts showed increased ASK1/p-p38 levels not only at resting state (Figure 3D) but also after cold storage (Figure 3E). Thus, CEACAM1 was essential to inhibit a cold stimulationtriggered ASK1/p-p38 signaling pathway.

Hepatocyte CC1 inhibits p-p38 upregulation and cell death under cold stress via the ASK1 pathway. Having demonstrated the hepatoprotective role of CEACAM1 in cold-stressed liver grafts (Figure 2, $\mathrm{B}$ and $\mathrm{C}$ ) and the inhibitory function of $C C 1$ against ASK1/p-p38 signaling (Figure 3E), we next asked whether the ASK1/p-p38 axis may be essential for CEACAM1-mediated hepatocyte protection. In a refined murine primary hepatocyte culture (WT), 4 hours of cold stimulation $\left(4^{\circ} \mathrm{C}\right)$ distinctly increased expression of ASK1 and p-p38 (Figure 4A), upregulated 4HNE expression (Figure 4C, upper panel), induced HMGB1 translocation to cytoplasm (Figure $4 \mathrm{C}$, middle panel), ultimately leading to hepatocyte death (Figure $4 \mathrm{C}$, lower panel, and Figure 4D). Notably, hepatocyte CC1 deficiency further enhanced ASK1/p-p38 (Figure 4B), 4HNE expression, HMGB1 translocation, and cell death (Figure 4, C and D). In contrast, ASK1 silencing using siRNA (ASK1) in cold-stressed CC1-deficient hepatocyte cultures inhibited p-p38 overexpression, 4HNE upregulation, HMGB1 translocation, and cell death (Figure 4, B-D). These data indicate the important contribution of ASK1 signaling in p-p38 regulation and CECAM1-mediated hepatocyte protection during cold stimulation.

ASK1 inhibition suppresses p-p38 upregulation, 4HNE overexpression, and HMGB1 translocation in cold-stressed CC1-deficient liver grafts. To verify in vivo relevance of the aforementioned in vitro findings (Figure 4), we next incubated CC1-KO livers with a selective ASK1 inhibitor (19) during 18 hours of cold storage. CC1-deficient liver grafts supplemented with ASK1 inhibitor showed suppressed p-p38 upregulation (Figure 5A), neither 4HNE overexpression nor cytoplasmic translocation of HMGB1 (Figure
5B), and also decreased HMGB1 secretion in the liver flush after cold stimulation (Figure 5C). Thus, liver CC1 deficiency increased p-p38/4HNE expression and promoted HMGB1 translocation/ tissue damage via ASK1 signaling.

ASK1 signal inhibition during cold storage alleviates hepatic IRI and improves CC1-KO OLT survival. To investigate the function of ASK1 in aggravated hepatic IR damage, we transplanted CC1-KO livers with or without preincubation with ASK1 inhibitor during cold storage into WT recipients. Addition of ASK1 inhibitor during cold stimulation $\left(4^{\circ} \mathrm{C} / 18\right.$ hours $)$ attenuated sinusoidal congestion, edema/vacuolization, and hepatocellular necrosis (Figure 5D), decreased sAST/ALT levels (sAST: CC1-KO $\rightarrow \mathrm{WT}=6035 \pm$ 1688 vs. CC1-KO+ASK1 inhibitor $\rightarrow \mathrm{WT}=3552 \pm 1083 \mathrm{IU} / \mathrm{L}, P=$ 0.0020; sALT: $\mathrm{CC} 1-\mathrm{KO} \rightarrow \mathrm{WT}=10573 \pm 2824$ vs. CC1-KO+ASK1 inhibitor $\rightarrow \mathrm{WT}=6744 \pm 2648, P=0.0271$; Figure $5 \mathrm{E}$ ), decreased Suzuki's histological grading of IRI (CC1-KO $\rightarrow \mathrm{WT}=6.3 \pm 1.0$ vs. $\mathrm{CC1}-\mathrm{KO}+\mathrm{ASK} 1$ inhibitor $\rightarrow \mathrm{WT}=2.0 \pm 1.2, P<0.0001$; Figure $5 \mathrm{~F}$ ), and suppressed frequency of TUNEL-positive cell death (CC1-KO $\rightarrow \mathrm{WT}=82.0 \pm 11.8$ vs. CC1-KO+ASK1 inhibitor $\rightarrow \mathrm{WT}=39.7 \pm$ $5.7 / \mathrm{HPF}, P<0.0001$; Figure 5, D and G) at 6 hours after reperfusion in otherwise IRI-susceptible CC1-KO grafts. Moreover, unlike recipients of CC1-KO grafts with poor prognosis and outcomes as compared with WT $\rightarrow$ WT pairs (20-day survival: 0\% versus $44 \%$, $P=0.0068$ ), incubation of donor livers with ASK1 inhibitor significantly improved overall survival of CC1-KO $\rightarrow$ WT hosts (50\% vs. $0 \%, P=0.0217$ ) (Figure $5 \mathrm{H}$ ). Thus, preincubation of CC1-KO livers with ASK1 inhibitor during cold storage mitigated hepatic IRI and improved otherwise inferior post-OLT survival.

CEACAM1 levels in cold-stressed human donor livers negatively correlate with $A S K 1 / p-p 38$ expression in OLT patients. Having demonstrated the regulatory CEACAM1 - ASK1/p-p38 crosstalk in cold-stimulated murine liver grafts, we next aimed to validate its relevance in clinical liver transplant patients. Sixty cold-stored human liver biopsies (Bx), collected at back table before implantation, were screened by Western blots for CEACAM1, ASK1, and p-p38 protein levels with $\beta$-actin normalization (Supplemental Figure 1A; supplemental material available online with this article; https://doi.org/10.1172/JCI133142DS1). Hepatic CEACAM1 correlated negatively with ASK1 $(r=-0.3424, P=0.0074$, Supplemental Figure 1B) and p-p38 expression $(r=-0.2947, P=0.0222$, Supplemental Figure 1C). Based on Western-assisted relative CEACAM1 expression pattern, pretransplant human Bx samples collected from 60 OLT patients were divided into low CEACAM1 $(n=30)$ and high CEACAM1 expression groups $(n=30)$, based on the relative CEACAM1 $/ \beta$-actin levels, according to median split method (cutoff $=$ 0.85 , Figure $6 \mathrm{~A}$ ). There was no correlation between CEACAM1 classification and recipient/surgical parameters, including age, sexual phenotype, race, BMI, disease etiology, ABO compatibility, model for end-stage liver disease (MELD) score, pretransplant blood tests, preoperative hospital stay, cold ischemia time (CIT), warm ischemia time (WIT), and blood transfusions during surgery (Supplemental Table 1). There was no correlation between CEACAM1 grouping and donor variables, such as age, sexual phenotype, race, BMI, preprocurement blood tests, and donation status (donation after circulatory death [DCD] or donation after brain death [DBD]) (Supplemental Table 2). Consistent with data in Supplemental Figure $1, \mathrm{~B}$ and $\mathrm{C}$, the low CEACAM liver transplant clinical cohort 
A

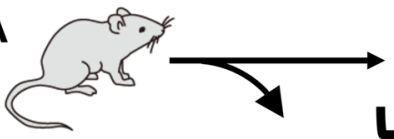

Donor

Naive

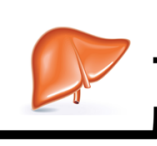

$18 \mathrm{~h}$ cold storage

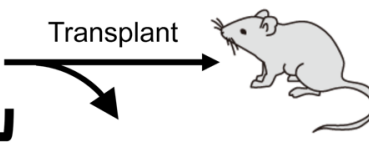

$18 \mathrm{~h}$ after Recipent cold storage

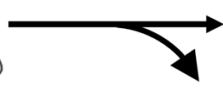

$3 \mathrm{~h}$ after reperfusion

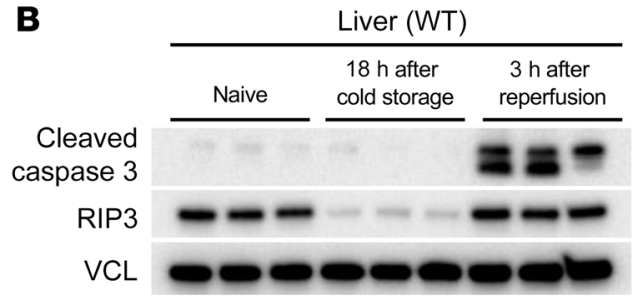

Cleaved caspase 3/VCL

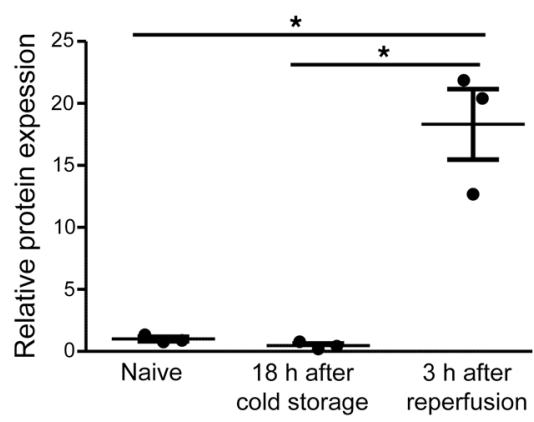

RIP3/VCL

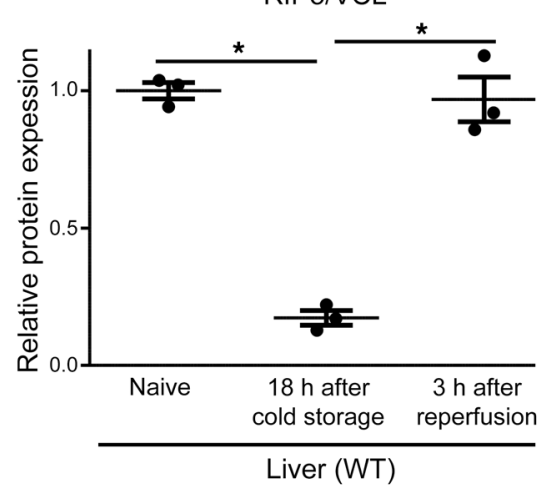

C

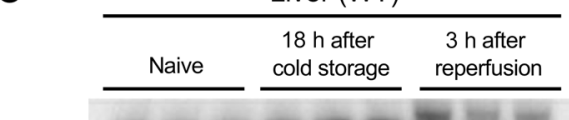

CC1

ASK1

p-p38

VCL

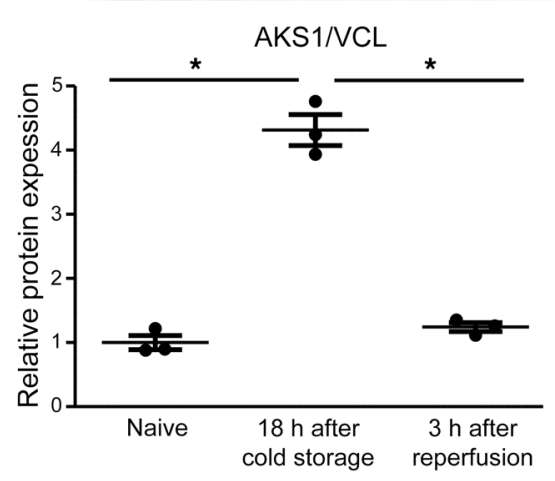

p-p38/VCL

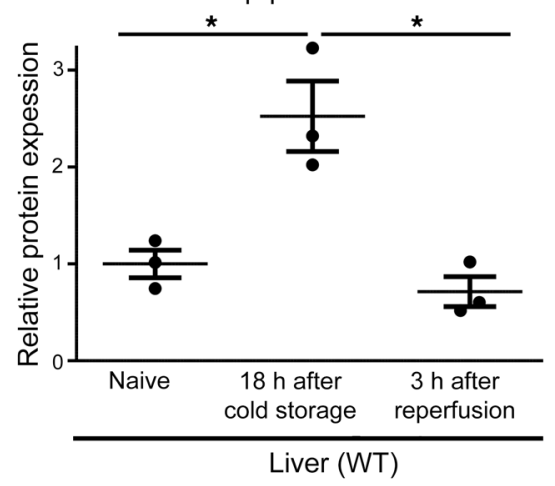

E

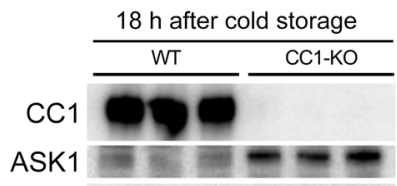

p-p38 - -

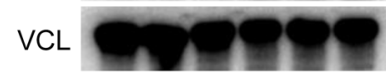

ASK1/VCL
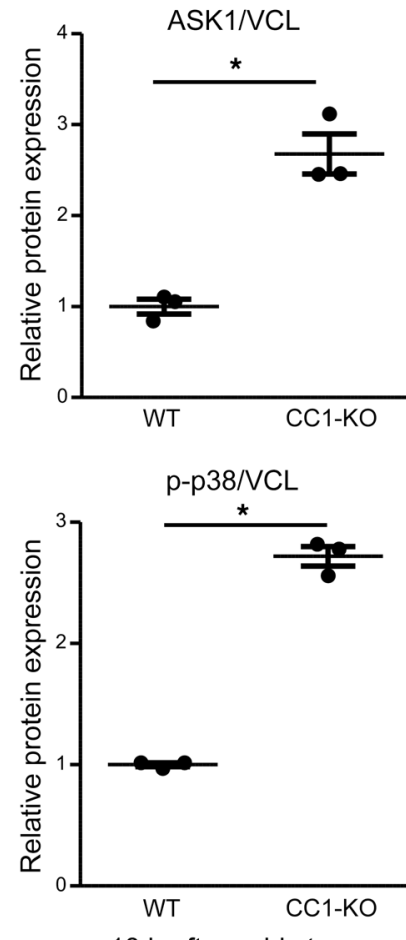

$18 \mathrm{~h}$ after cold storage

D

ASK1/VCL
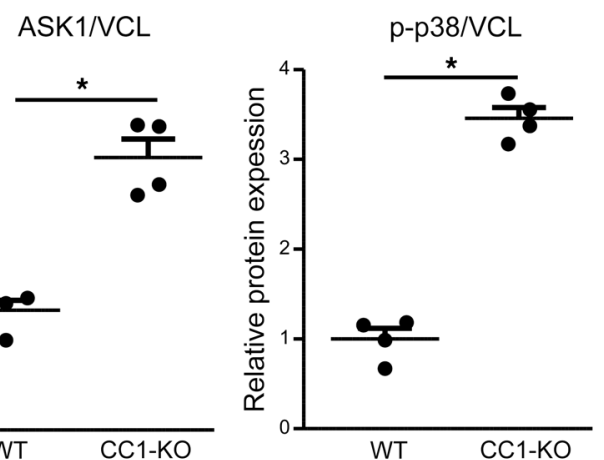

Figure 3. Donor hepatic CC1 deletion enhances cold stress-triggered ASK1/p-p38 signaling. (A) Groups of WT and CC1-KO livers were stored in UW solution $\left(4^{\circ} \mathrm{C} / 18\right.$ hours). Liver samples were collected right after cold storage (before OLT) or 3 hours after reperfusion (post-OLT). (B and C) Western blot-assisted detection and relative intensity ratio of cleaved caspase-3, RIP3, CC1, ASK1, p-p38 in naive liver, cold-stored liver or postreperfusion OLT (WT). Vinculin (VCL) expression served as an internal control and was used for normalization ( $n=3 /$ group). (D) Western blot-assisted detection and relative intensity ratio of CC1, ASK1, and p-p38 in naive WT or CC1-KO liver. VCL expression served as an internal control and was used for normalization ( $n=4 / g r o u p)$. (E) Western blotassisted detection and relative intensity ratio of CC1, ASK1, and p-p38 in cold-stored WT or CC1-KO livers. VCL expression served as an internal control and used for normalization ( $n=3$ /group). Data shown as mean \pm SD. ${ }^{*} P<0.05$, 1-way ANOVA followed by Tukey's HSD test (B and $\left.\mathbf{C}\right)$ or Student's $t$ test (D and E). 
A

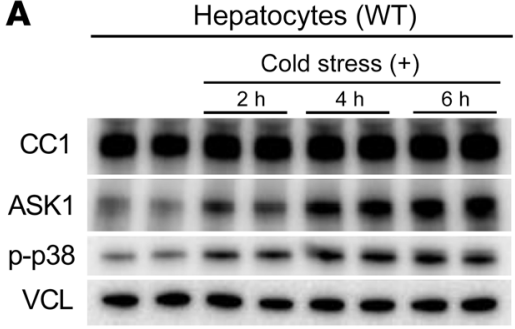

B

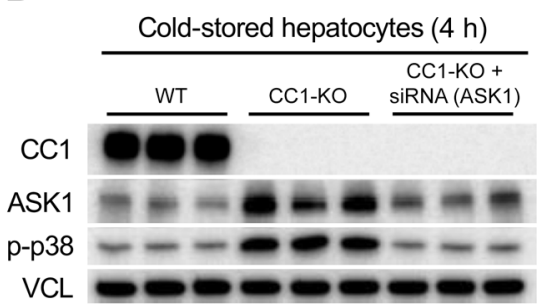

Cold-stored hepatocytes (WT)

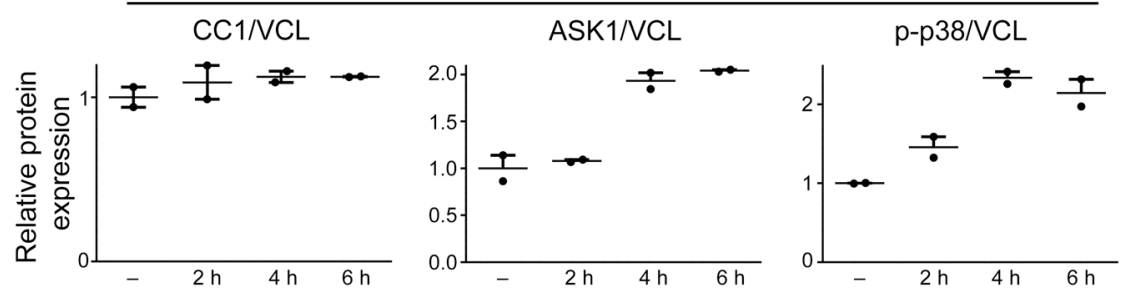

Cold-stored hepatocytes $(4 \mathrm{~h})$

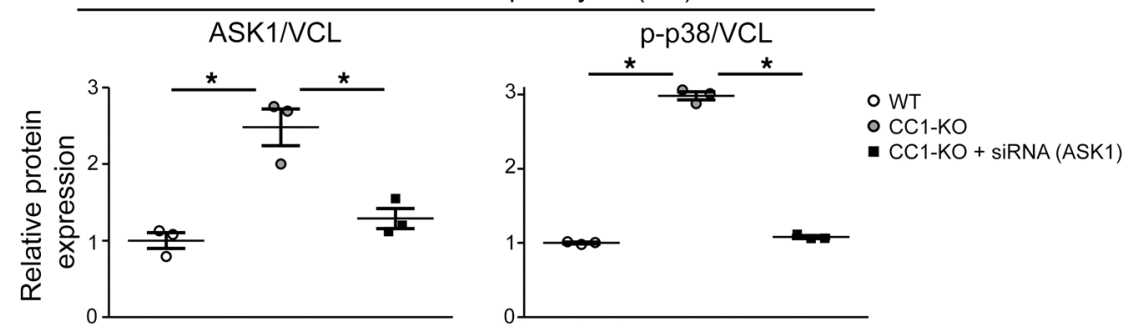

C
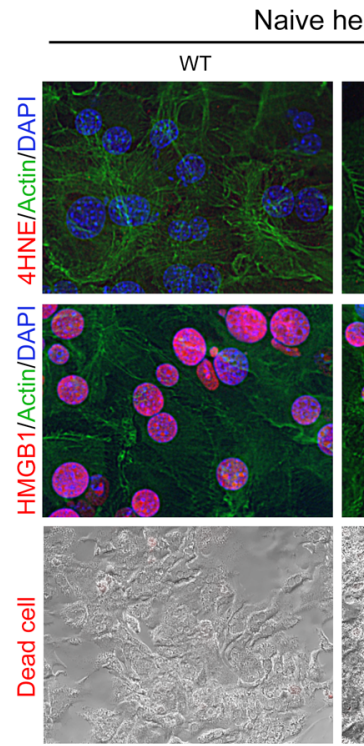

epatocytes
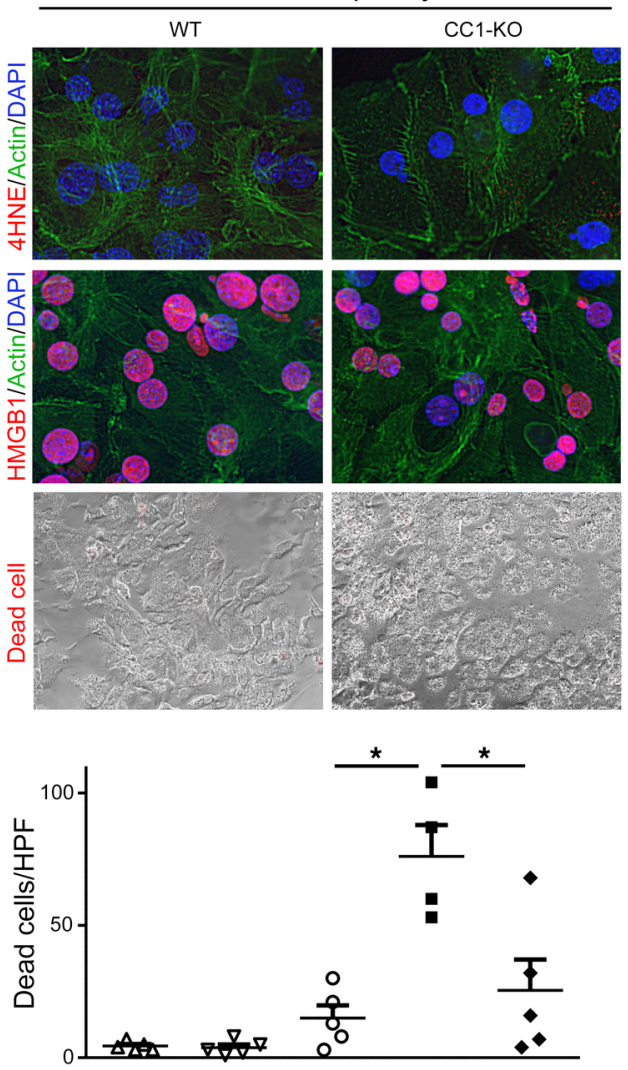

Cold-stored (4 h) hepatocytes
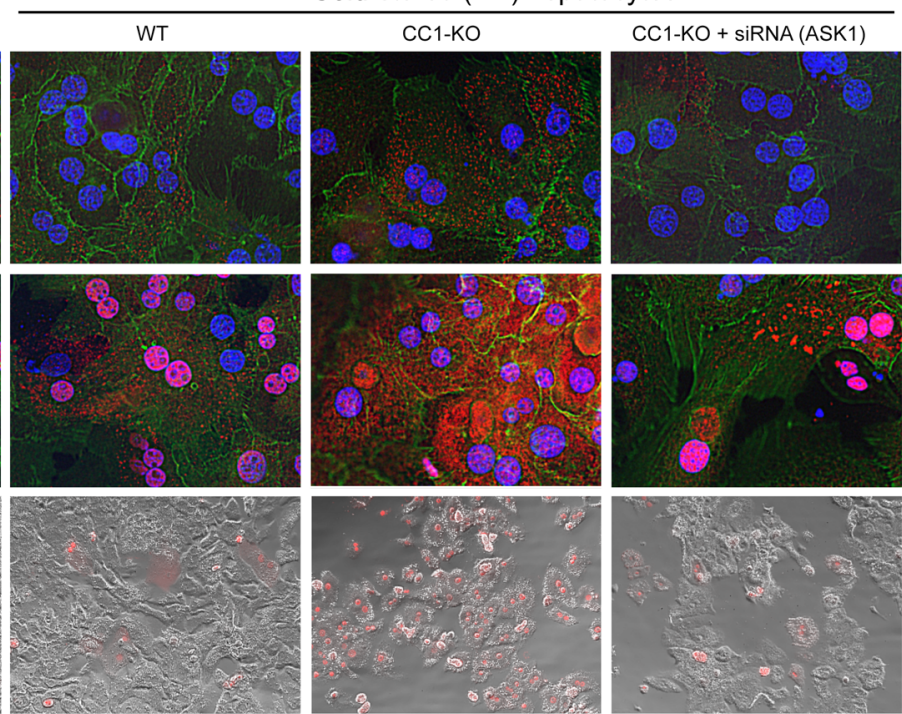

$\Delta$ Naive hepatocytes: WT

$\boldsymbol{\nabla}$ Naive hepatocytes: CC1-KO

O Cold-stored ( $4 \mathrm{~h}$ ) hepatocytes: WT

Cold-stored (4 h) hepatocytes: CC1-KO

- Cold-stored (4 h) hepatocytes: CC1-KO + siRNA (ASK1)

Figure 4. Hepatocyte CC1 deficiency enhances p-p38 increase, 4HNE overexpression, HMGB1 translocation, and cell death due to cold stress in an ASK1-dependent manner. (A) Primary mouse hepatocytes (WT) with or without cold stimulation $\left(4^{\circ} \mathrm{C} / 4\right.$ hours) were incubated for the indicated time periods. Western blot-assisted detection and relative intensity ratio of CC1, ASK1, p-p38. VCL expression served as an internal control and used for normalization ( $n=2$ /group). (B-D) Cold-stimulated WT or CC1-KO hepatocytes were pretreated with or without siRNA against ASK1. (B) Western blot-assisted detection and relative intensity ratio of CC1, ASK1, p-p38. VCL expression served as an internal control and used for normalization ( $n=3 /$ group). (C) Representative ( $n=3 /$ group) immunohistochemical staining of 4HNE (red, upper panels), HMCB1 (red, middle panels), and dead cell detection (red, lower panels). (D) Quantification of dead cells/HPF ( $n=4-5 /$ group). ${ }^{*} P<0.05,1$-way ANOVA followed by Tukey's HSD test.

showed higher ASK1 $(P=0.0459)$ and p-p38 $(P=0.0153)$ levels as compared with high CEACAM1 cases (Figure 6B). Representative Western blots are shown in Figure 6C. Consistent with mouse OLT data (Figure 2C), low CEACAM1 levels in clinical liver Bx samples were associated with increased 4HNE (Figure 6D) and enhanced cytoplasmic HMGB1 translocation (Figure 6E).

CEACAM1 levels before liver implantation associate with the hepatocellular function in human OLT recipients. Since disruption of 
A

A Cold-stored (18 h) liver graft

\section{WT CC1-KO $\begin{gathered}\text { CC1-KO+ } \\ \text { ASK1 inhibitor }\end{gathered}$ \\ cc1 \\ p-p38 - - - \\ VCL}

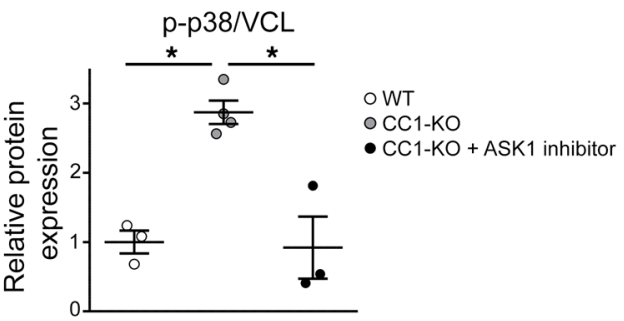

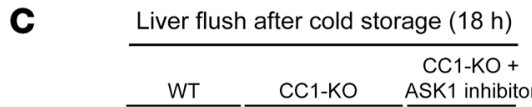

HMGB1 $\frac{\ldots}{20 \mu \mathrm{L} \text { of flush }}$

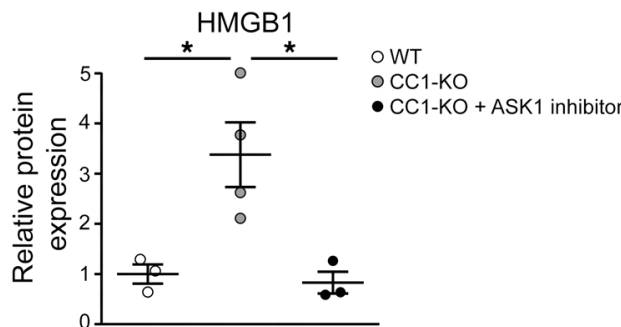

D

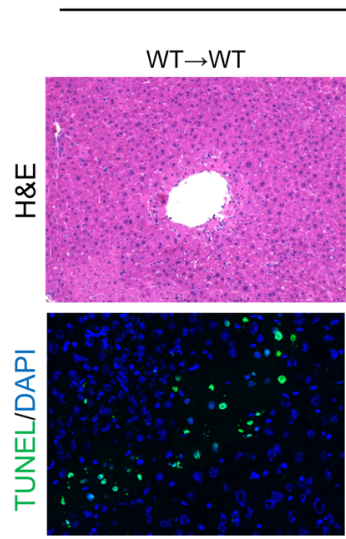

$6 \mathrm{~h}$ after OLT

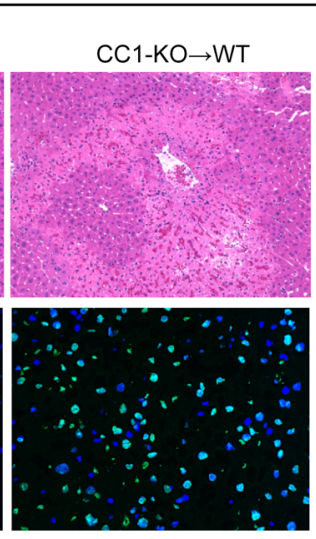

$6 \mathrm{~h}$ after OLT

$\mathbf{F}$

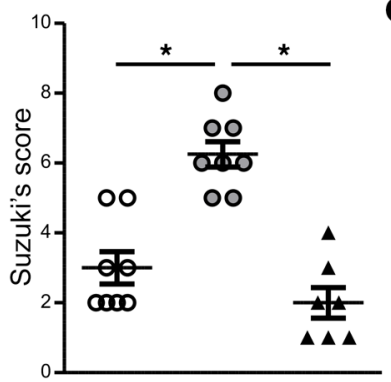

B

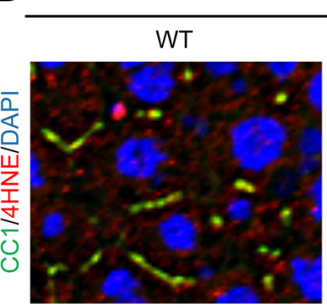

Cold-stored (18 h) liver graft
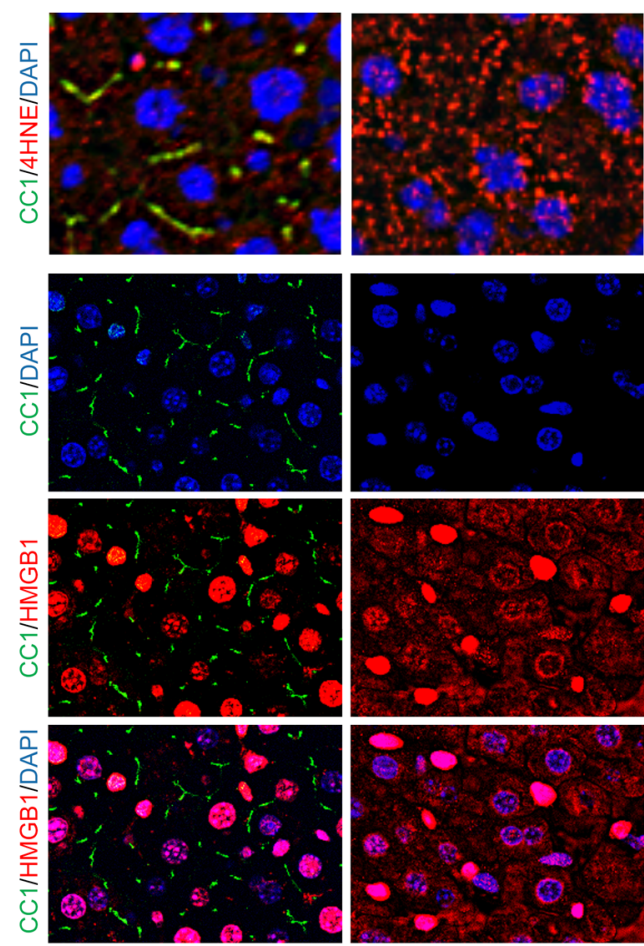

CC1-KO + ASK1 inhibitor
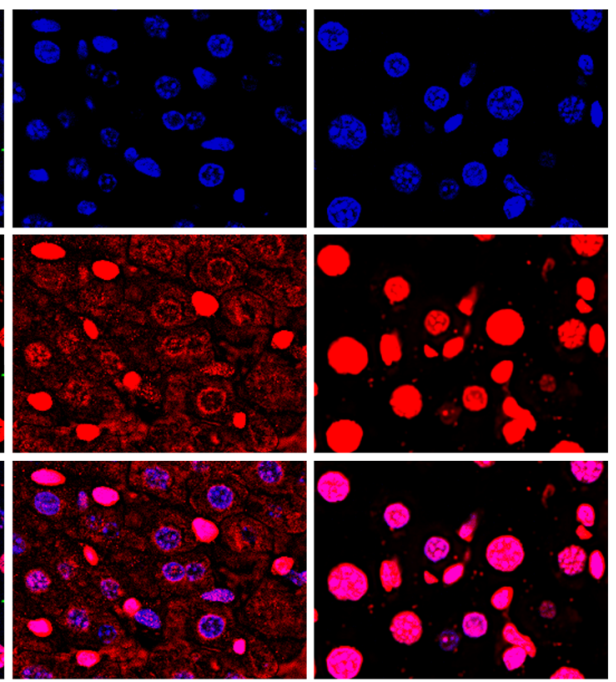

E

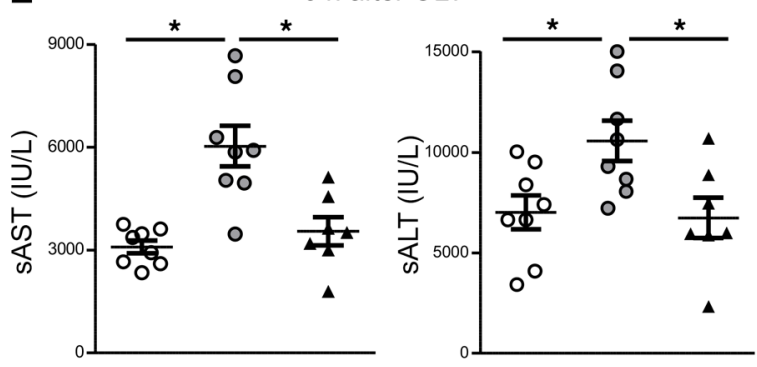

O $\mathrm{WT} \rightarrow \mathrm{WT}$

- $\mathrm{CC} 1-\mathrm{KO} \rightarrow \mathrm{WT}$

a CC1-KO + ASK1 inhibitor $\rightarrow$ WT

H Mouse survival after OLT

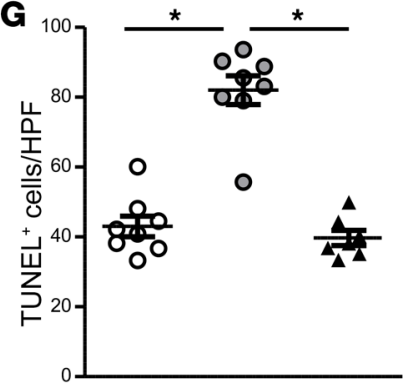

O $\mathrm{WT} \rightarrow \mathrm{WT}$

- $\mathrm{CC} 1-\mathrm{KO} \rightarrow \mathrm{WT}$

CC1-KO + ASK1 inhibitor $\rightarrow$ WT

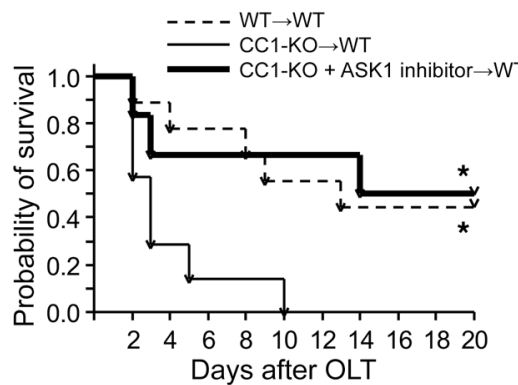


Figure 5. Inhibition of ASK1 in CC1-KO livers prevents cold-triggered p-p38 increase, 4HNE overexpression, and HMGB1 translocation as well as OLT damage and recipient mortality. (A-C) Groups of WT and CC1-KO livers were stored in UW solution $\left(4^{\circ} \mathrm{C} / 18\right.$ hours) with or without $\mathrm{ASK} 1$ inhibitor $(10 \mu \mathrm{g} / 15 \mathrm{~mL})$. (A) Western blot-assisted detection and relative intensity ratio of $\mathrm{CC} 1$ and $\mathrm{p}-\mathrm{p} 38$. VCL expression served as an internal control and used for normalization ( $n=3-4$ /group). (B) Representative ( $n=$ 3/group) immunohistochemical staining of CC1/4HNE and CC1/HMGB1. (C) Liver flush $(20 \mu \mathrm{L})$ from cold-stressed WT or CC1-KO livers with or without ASK1 inhibitor were analyzed by Western blots for HMGB1 levels ( $n=3-4$ / group). (D-H) Cold-stored ( $4^{\circ} \mathrm{C} / 18$ hours) WT or CC1-KO livers were transplanted into recipient mice, and OLT and serum samples were analyzed at 6 hours after reperfusion. Some CC1-KO grafts were preincubated with ASK1 inhibitor $(10 \mu \mathrm{g} / 15 \mathrm{~mL})$ during cold storage $\left(4^{\circ} \mathrm{C} / 18\right.$ hours). Separate OLT recipient groups were monitored for 20-day survival. (D) Representative $H \& E$ (original magnification $\times 100$ ) and TUNEL staining. (E) SAST and sALT levels (IU/L; $n=7-8 /$ group). (F) Suzuki's histological grading of liver IRI ( $n=7-8$ /group). (G) Quantification of TUNEL-positive cells/HPF ( $n=$ 7 -8/group). Data shown as mean \pm SD. ${ }^{*} P<0.05$, 1-way ANOVA followed by Tukey's HSD test. (H) Recipient mice were monitored for 20 days and cumulative survival was analyzed (Kaplan-Meier method). Dotted line: WT $\rightarrow \mathrm{WT}$; solid line: $\mathrm{CC1}$-KO $\rightarrow \mathrm{WT}$; bold line: $\mathrm{CC} 1-\mathrm{KO}+\mathrm{ASK} 1$ inhibitor $\rightarrow \mathrm{WT}$ ( $n=6$-9/group; ${ }^{*} P<0.05$ vs. CC1-KO $\rightarrow$ WT, log-rank test).

liver CEACAM1 signaling in mouse OLT not only affected stress resistance under cold storage (Figures 2 and 3) but was also critical for postreperfusion injury and recovery (Figure 1), we next aimed to evaluate correlation between pretransplant CEACAM1 levels and postreperfusion hepatic damage in human OLT. Pretransplant CEACAM1 expression correlated negatively with sAST $(r=-0.3302, P=0.0100$, Supplemental Figure 1D) and sALT $(r=$ $-0.3280, P=0.0105$, Supplemental Figure 1E) levels at postoperative day 1 (POD1). In addition, low CEACAM1 cases (Figure 6A) exhibited significantly higher sAST at POD1-5 (Figure 7A) and sALT at POD1-7 (Figure 7B). We then analyzed liver Bx samples obtained at 2 hours after reperfusion (before the abdominal closure) from the corresponding low vs. high CEACAM1 clinical cases. The TUNEL staining from representative postreperfusion Bx samples is shown (Figure 7C, low CEACAM1: $133.8 \pm 26.0$ /HPF; high CEACAM1: $69.8 \pm 24.23 / \mathrm{HPF} ; P=0.0611, n=4$ /group). Pretransplant low CEACAM1 livers exhibited increased mRNA levels coding for TLR4 $(P=0.0065), \operatorname{CD} 80(P=0.0910), \operatorname{CD} 86(P=0.0112)$, CXCL10 $(P=0.0866)$, CD68 $(P=0.1004)$ (macrophage activation markers); Cathepsin G $(P=0.0052)$ (neutrophil marker); CD28 $(P=0.0189), \mathrm{CD} 4(P=0.0346)$, IL17 $(P=0.0327)$ (T cell markers) in OLTs at 2 hours after reperfusion (Figure 8). Thus, pretransplant low CEACAM1 expression was associated with enhanced postreperfusion innate/adaptive immune responses and increased hepatocellular damage in the early post-OLT period. Although low CEACAM1 cases experienced increased frequency of EAD as compared with the high CEACAM1 group (30.0\% vs. 16.7\%), the differences failed to reach statistical significance $(P=0.3604$, Figure 7D). To examine the relationship between pretransplant CEACAM1 expression and OLT outcomes, we analyzed the overall graft survival (median follow-up, 1269 days; range, 3-1892 days) and rejection-free graft survival (median follow-up, 1249 days; range, 3-1892 days). None of the patients underwent secondary liver transplantation. Despite obvious trends, inferior graft survival $(P=0.3293$, Figure 7E) and rejection-free graft survival $(P=0.1674$,
Figure 7F) in the low CEACAM1 group failed to reach statistical significance when compared with the high CEACAM1 group.

CEACAM1 expression before liver implantation dictates the incidence of EAD in human OLT recipients. Having failed to establish a statistical correlation between the EAD rate and pretransplant CEACAM1 expression level when the median level (CEACAM1/ $\beta$-actin: 0.85) was simply employed as a cutoff (Figure 6A and Figure 7D), we next aimed to seek the optimal cutoff to evaluate predictive ability of pretransplant CEACAM1 for EAD. Based on a ROC curve and Youden index on the basis of best accuracy in relation to an incidence of EAD, the optimal pretransplant CEACAM1/ $\beta$-actin cutoff values were selected by maximizing sum of sensitivity and specificity $(0.71$, AUROC $=0.604$, sensitivity $=0.761$, specificity $=0.643$ ) (Figure 9A and Supplemental Table $3)$. Based on the cutoff value of CEACAM1/ $\beta$-actin being 0.71 , 60 OLT clinical cases were classified into two groups: CEACAM1/ $\beta$-actin less than $0.71(n=20)$ and CEACAM1/ $\beta$-actin greater than $0.71(n=40)$ (Figure 9B). There was no correlation between CEACAM1 classification and recipient age, sexual phenotype, BMI, disease etiology, ABO compatibility, MELD score, pretransplant blood tests, CIT, WIT, and blood transfusion required for OLT operations, whereas we found statistically significant differences in recipient race and preoperative hospital stay (Supplemental Table 4). There was no correlation between CEACAM1 grouping and donor variables, including age, sexual phenotype, race, BMI, preprocurement blood tests, and DCD (Supplemental Table 5). Likewise to the data shown in Figure 7, A and B, the CEACAM1/ $\beta$-actin less than 0.71 group showed increased sAST levels at POD1, 2, 4, and 5, and higher sALT levels at POD1-7 (Figure 9C). Of note, the CEACAM1 $/ \beta$-actin less than 0.71 cases experienced a significantly increased incidence of EAD as compared with the CEACAM $1 / \beta$-actin greater than 0.71 cases $(45.0 \%$ vs. $12.5 \%, P=$ 0.0088 , Figure 9B). To determine whether a CEACAM $1 / \beta$-actin level less than 0.71 represents an independent predictor of EAD in our liver transplant patient cohort, we conducted multivariate analysis based on a stepwise logistic regression model by screening the phrase "pre-OLT CEACAM1/ $\beta$-actin $<0.71$ " simultaneously with recipient age ( $\geq 60$ years), recipient sexual phenotype (male), recipient race, recipient BMI $\left(\geq 25 \mathrm{~kg} / \mathrm{m}^{2}\right)$, preoperative hospital stay ( $\geq 7$ days), disease etiology, MELD score ( $\geq 35)$, cold ischemia time ( $\geq 420$ minutes), warm ischemia time ( $\geq 50 \mathrm{~min}$ utes), donor age ( $\geq 40$ years), donor sexual phenotype (male), donor BMI $\left(\geq 25 \mathrm{~kg} / \mathrm{m}^{2}\right)$, and DCD donor. Remarkably, "pre-OLT CEACAM1 $/ \beta$-actin $<0.71$ " was identified as one of the predictive factors of $\mathrm{EAD}$ (OR $=7.209$ [95\% CI: 1.376-37.755], $P=0.019$ ), along with cold ischemia time ( $\geq 420$ minutes) $(\mathrm{OR}=13.024$ [95\% CI: 1.873-90.551], $P=0.009$ ) (Figure 9D). Despite obvious trends of inferior graft survival $(P=0.3756$, Supplemental Figure 2A) and rejection-free graft survival ( $P=0.4093$, Supplemental Figure 2B), the patient group with pre-OLT CEACAM1/ $\beta$-actin less than 0.71 failed to reach statistical significance when compared with the patient group with pre-OLT CEACAM1/ $\beta$-actin greater than 0.71 .

\section{Discussion}

Although the expression of CEACAM1 cell-surface glycoprotein was originally detected on rat hepatocellular membranes $(12,13)$, a growing body of evidence is now linking its function with meta- 

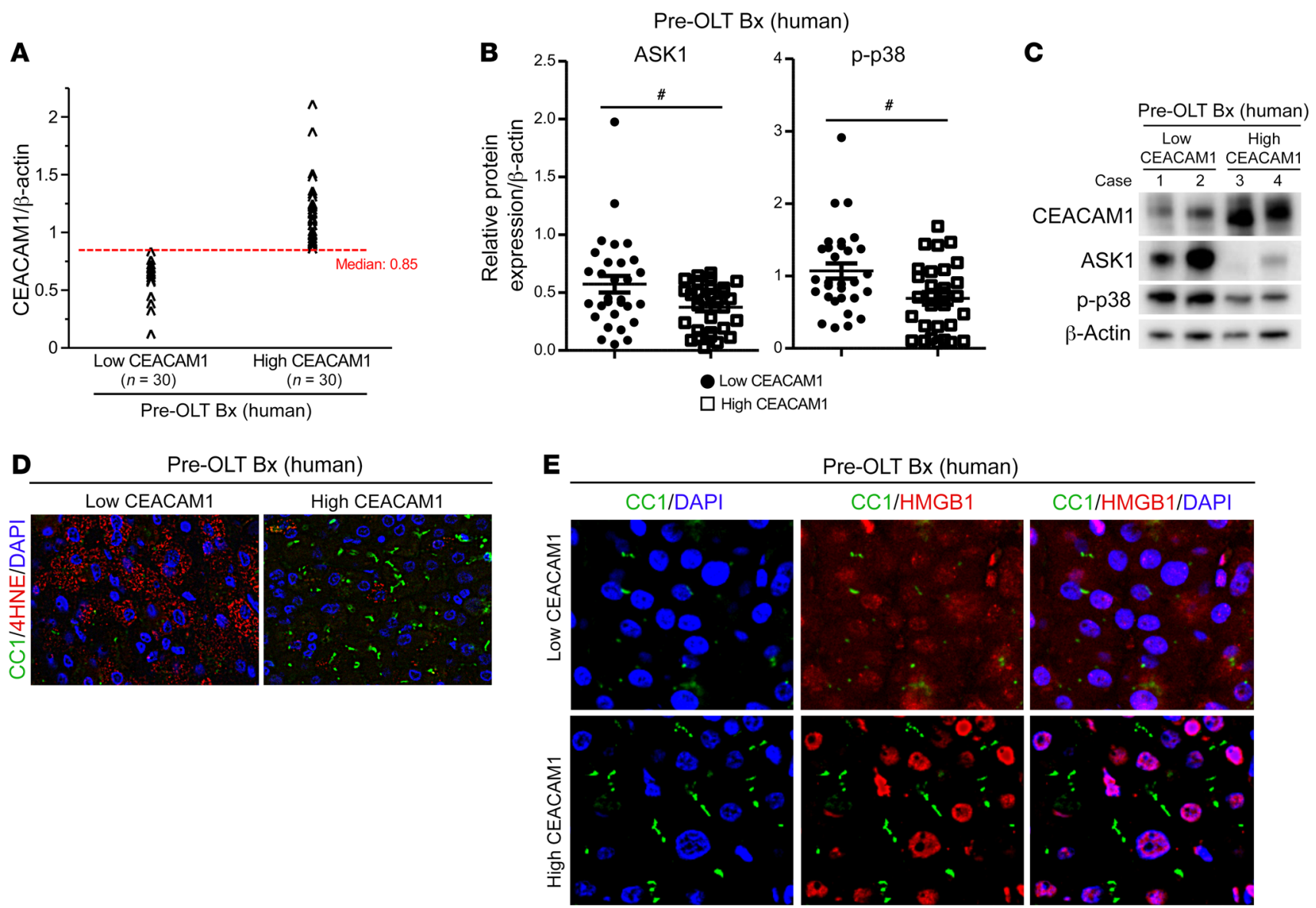

Figure 6. Pretransplant CEACAM1 levels are associated with ASK1/p-p38/4HNE expression and HMGB1 translocation in human OLT recipients. Pretransplant (after cold storage) human liver Bx $(n=60)$ were analyzed by Western blots with $\beta$-actin normalization for CEACAM1, ASK1, and p-p38 levels (see Supplemental Figure 1A). (A) Bx samples were divided into low $(n=30)$ and high $(n=30)$ CEACAM1 expression groups based on the relative CEACAM1/ $\beta$-actin levels (cutoff $=0.85$, median). (B) Western blot-assisted expression of ASK1 and $p$-p38. Data shown in dot plots and bars indicate mean \pm SEM. ${ }^{\#} P<0.05$ (Mann-Whitney $U$ test). (C) Four representative Western blots are shown (case 1/2: low CEACAM1, case 3/4: high CEACAM1). (D) Representative $(n=3)$ CEACAM1/4HNE staining (original magnification $\times 200)$. (E) Representative $(n=3)$ CEACAM1/HMCB1 staining (original magnification $\times 400)$.

bolic disorders, such as hepatic insulin clearance and NASH (20). By focusing on CEACAM1 regulation in T cell activation, Horst et al. reported the aggravated and persistent hepatocellular damage in CC1-deficient mice subjected to Con A-induced T cell-mediated liver injury (20). Despite being the principal CEACAM1expressing liver cell type, whether and how hepatocyte CEACAM1 may contribute to stress resistance remains largely unknown. Our report identifies what we believe to be novel cytoprotective functions of and mechanisms by which hepatic CEACAM1 signaling controls IR stress in mouse and human liver transplantation.

In the experimental arm, in a clinically relevant mouse OLT model with extended (18 hours) cold storage, graft CC1 null mutation exacerbated hepatic sterile IR inflammation, and deteriorated the hepatocellular function in WT recipients. This was manifested by enhanced histopathology tissue injury scores, increased release of sAST/sALT enzymes, augmented TUNEL-positive hepatocellular death rate accompanied by enhanced HMGB1 release, along with leukocyte trafficking and proinflammatory gene expression profiles (Figure 1). Of note, our in vivo and in vitro experiments document that CEACAM1 alleviated liver/hepatocyte damage in the early cold preservation phase, whereas its deficiency enhanced
4HNE expression (Figure 2C and Figure 4C), augmented HMGB1 cytoplasmic translocation (Figure 2C and Figure 4C), increased HMGB1 release into the liver flush (Figure 2B), and heightened hepatocyte death program (Figure 4C).

In the clinical arm consisting of 60 human liver transplant recipients, low CEACAM1 expression (CEACAM1/ $\beta$-actin less than the median of $0.85, n=30$ ) in liver grafts before the implantation (Figure 6A) was associated with increased post-OLT sAST/ sALT levels (Figure 7, A and B), as compared with grafts with high CEACAM1 expression (CEACAM1/ $\beta$-actin greater than the median of $0.85, n=30$ ), supporting the clinical relevance of CEACAM1-mediated hepatoprotection. Having failed to establish statistical correlation between EAD rate and pretransplant CEACAM1 when its median level was used as a cutoff, we established the optimized cutoff (CEACAM1 $\beta$-actin $=0.71$, Figure $9 A)$. Indeed, cold-stressed human liver grafts with CEACAM1/ $\beta$-actin less than 0.71 experienced significantly more frequent incidence of EAD than grafts with CEACAM1/ $\beta$-actin greater than 0.71 (45.0\% vs. $12.5 \%, P=0.0088$, Figure $9 \mathrm{~B}$ ). In addition, considering our group classification (CEACAM1/ $\beta$-actin less than 0.71 vs. CEACAM1/ $\beta$-actin greater than 0.71 ) may have been biased 
A

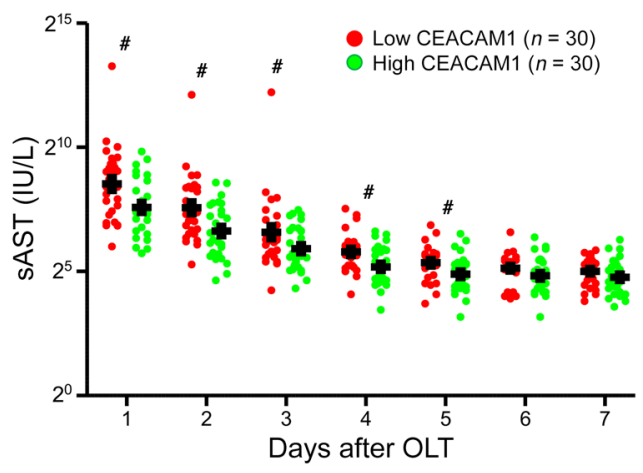

B

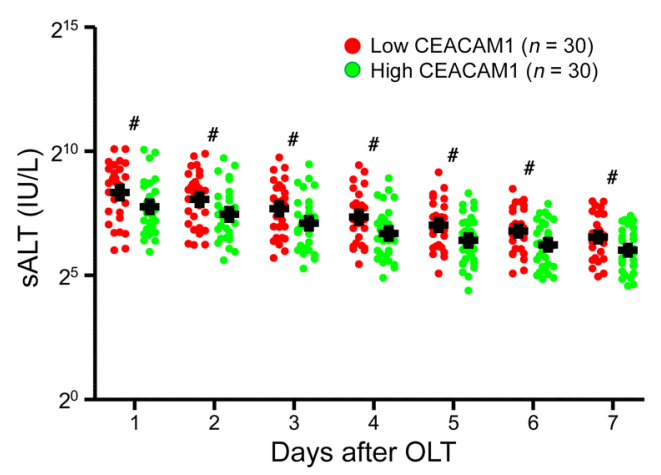

C Post-OLT Bx (human)

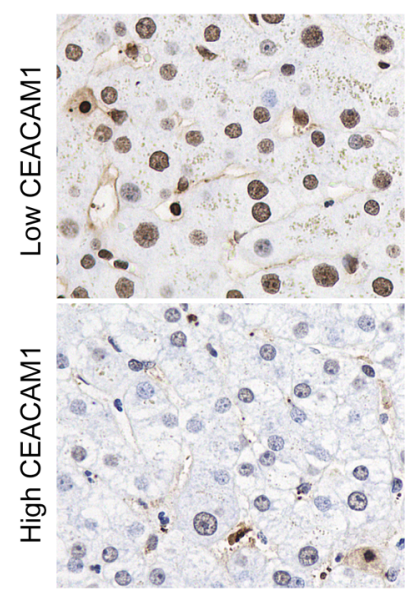

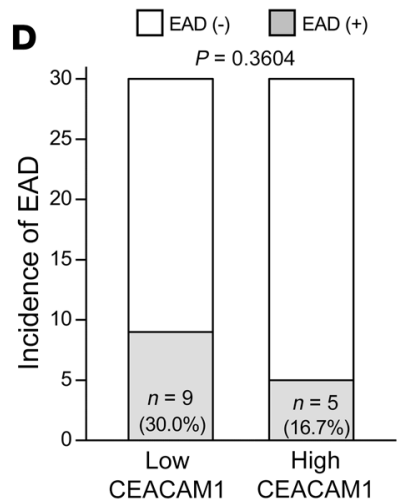
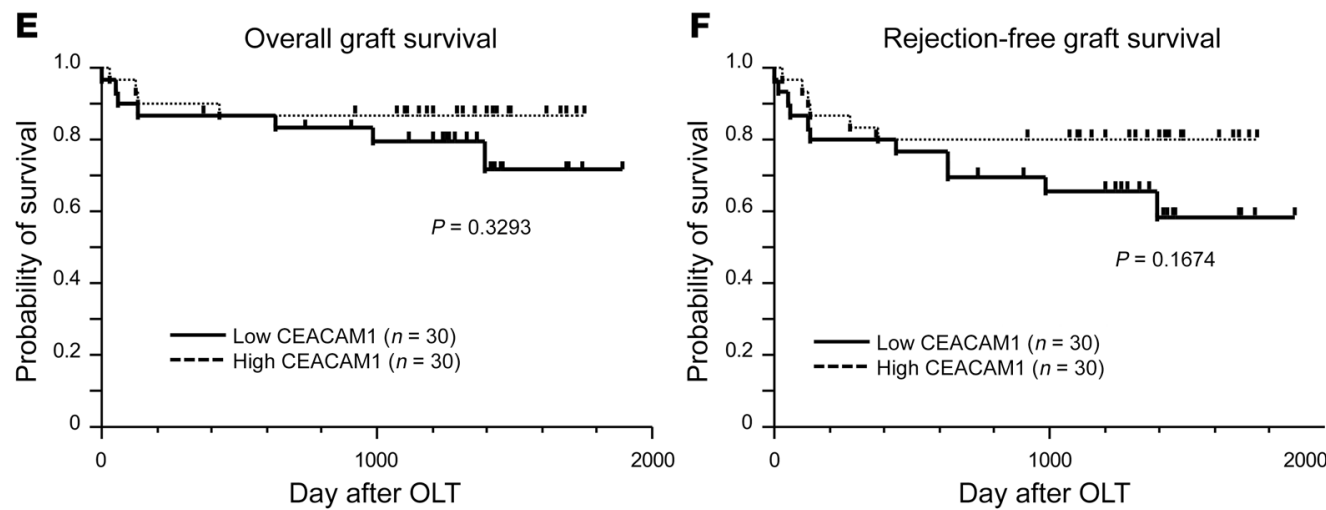

Figure 7. Low CEACAM1 levels impair hepatocellular function in human OLT recipients. Pretransplant (after cold storage) human liver BX samples were divided into low $(n=30)$ and high $(n=30)$ CEACAM1 expression groups, based on the relative CEACAM1/ $\beta$-actin levels (see Figure 6A). (A) Serum AST levels at POD1-7. (B) Serum ALT levels at P0D1-7. Data are mean \pm SEM. ${ }^{P} P<0.05$ (Mann-Whitney $U$ test). (C) Post-OLT Bx were obtained at 2 hours after reperfusion from corresponding clinical cases. Representative $(n=3)$ TUNEL staining (original magnification $\times 400)$. (D) Incidence of EAD (Fisher's exact test). (E) The cumulative probability of overall graft survival. (F) The cumulative probability of rejection-free graft survival. Solid line indicates low CEACAM1; dotted line indicates high CEACAM1 human OLT patient group (Kaplan-Meier method, log-rank test).

in terms of recipient preoperative variables (Supplemental Table 4), we conducted a multivariate analysis of the patient cohort to estimate the independent influence of graft CEACAM1 before implantation upon EAD rate by simultaneously screening putative prognostic factors and the biased recipient demographic variables (race and preoperative hospital stay). Remarkably, we identified CEACAM1 $/ \beta$-actin less than 0.71 as one of the independent predictors of EAD (OR = 7.209), together with cold ischemia time $(\geq 420$ minutes; $\mathrm{OR}=13.024$ ) (Figure 9D).

CEACAM1 signals through long cytoplasmic tail (CEACAM1-L), which provides inhibitory functions to different receptors (21), or through short cytoplasmic tail (CEACAM1-S) of less defined function, which may regulate mucosal immunity (22). Our ongoing studies suggest that IR-stressed hepatocytes/sinusoidal endothelial cells are enriched in CEACAM1-S isoform, whereas neutrophils/BMDM express predominantly CEACAM1-L. Indeed, in the present study (Figure 3C), naive and cold-stimulated mouse livers preferentially expressed CEACAM1-S (lower bands), whereas CEACAM1-L splicing variant (upper bands) was detected after reperfusion. Thus, tissue-resident CEACAM1-S may be key to mitigating cold-induced early hepatocellular dam- age, whereas CEACAM1-L is confined to OLT-infiltrating recipient immune cells after revascularization. As liver-sequestered Ly6G-positive neutrophils preferentially express CEACAM1-L isoform at 6 hours after reperfusion, while neutrophils express robust cytoplasmic $C C 1$ after reperfusion (unpublished observations), the putative role of CEACAM1-L in neutrophil regulation in IRI-OLT awaits future studies.

ASK1, a redox-sensitive upstream activator of the JNK and $\mathrm{p} 38$ arms of the MAPK pathway (23), may be activated by ROS, TNF- $\alpha$, ER stress, UV irradiation, or calcium overload, placing ASK1 as a signaling node in which different stressors converge (24-27). An increasing number of studies focus on the role of ASK1 in the pathophysiology of NASH (27), whereas the efficacy and tolerability of an ASK1 inhibitor, selonsertib, has been reported in a phase 2, randomized, open label clinical trial in patients with NASH (28). A recent in vitro study has also demonstrated the contribution of ASK1/p-p38 signaling axis in cell type-specific death under cold stress (11). In our mouse IRI-OLT and primary hepatocyte culture systems, cold stress triggered increased ASK1/p-p38 expression, whereas CEACAM1 signaling exerted potent hepatoprotection by suppressing the ASK1/p-p38 pathway (Figures 3-5). In support for 
Post-OLT Bx (human)
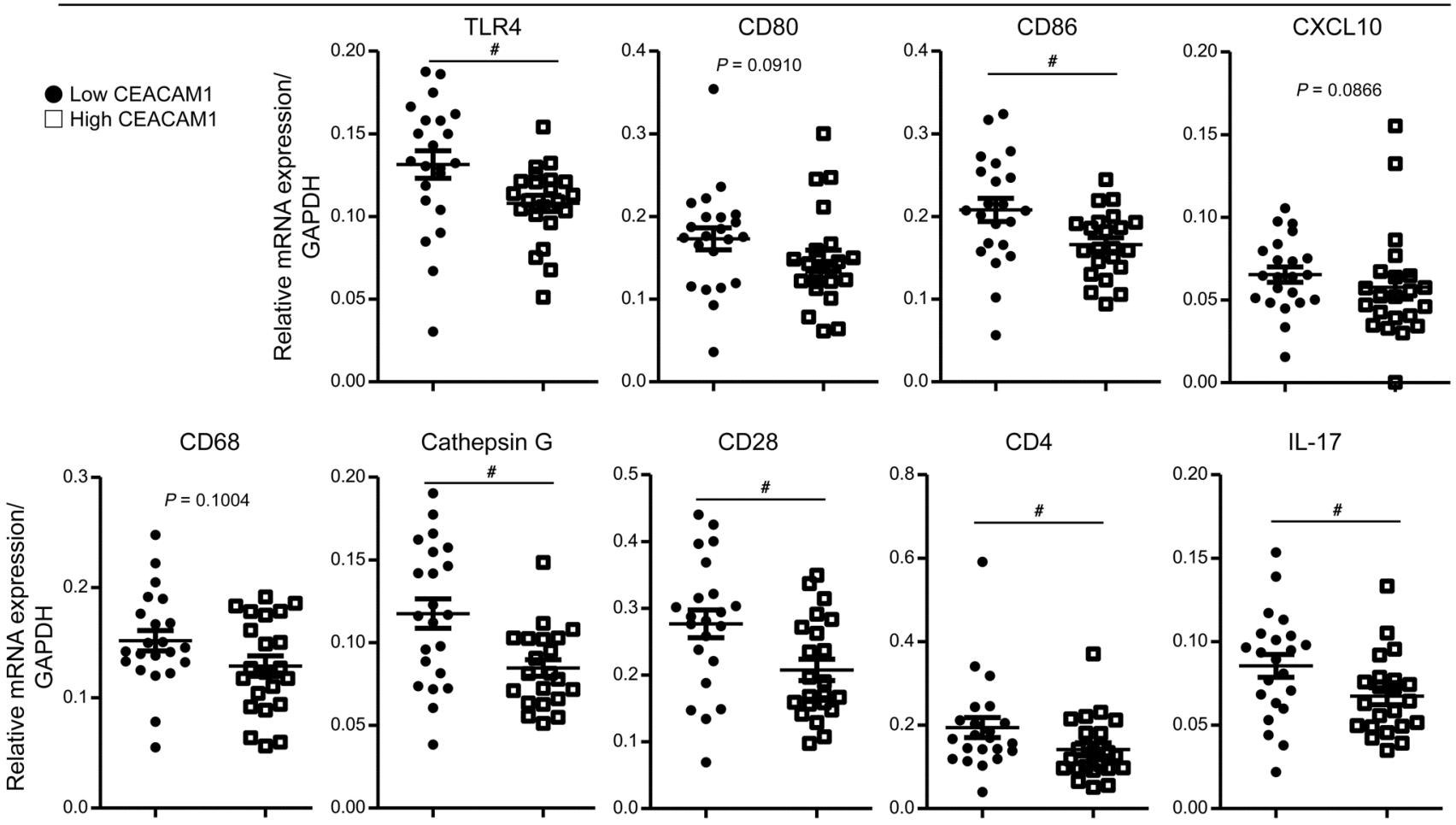

Figure 8. Depressed innate and adaptive gene activation pattern in human OLT is accompanied by high pretransplant hepatic CECAM1 levels. Pretransplant (after cold storage) human liver Bx samples were classified into low $(n=30)$ and high $(n=30)$ CEACAM1 (CC1) expression groups (see Figure 6A for details). Post-OLT Bx were obtained at 2 hours after reperfusion from corresponding cases, followed by qRT-PCR-assisted detection of mRNA coding for TLR4, CD80, CD86, CXCL10, CD68, Cathepsin G, CD28, CD4, and IL17. Data normalized to GAPDH gene expression are shown in dot plots and bars indicative of mean \pm SEM. ${ }^{\#} P<0.05$ (Mann-Whitney $U$ test).

hepatic CEACAM1 regulation of ASK1/p-p38 axis in humans, OLTs with low CEACAM1 expression consistently exhibited increased ASK1/p-p38 levels. Since adjunctive disruption of ASK1 signaling alleviated liver damage in otherwise highly IRI-susceptible CC1KO murine grafts (Figure 5), pretransplant ASK1 inhibition in low CEACAM1-expressing human livers may be considered.

Sustained cold stimulation evoked an increased ASK1/p-p38 expression pattern in mouse primary hepatocyte cultures and in donor livers without affecting CEACAM1 levels (Figure 3C and Figure 4A). In clinical pretransplant liver Bx samples, CEACAM1 expression correlated negatively with ASK1/p-p38 (Supplemental Figure 1, B and C), whereas CIT failed to show meaningful association with ASK1 $(r=-0.1038, P=0.4383)$ or p-p38 $(r=-0.0838$, $P=0.5317)$. Although these weak statistical correlations might be due to a limited number of study subjects, nevertheless, significant negative correlation between CEACAM1 and ASK1 $(r=-0.3424$, $P=0.0074$, Supplemental Figure 1B) or p-p38 $(r=-0.2947, P=$ 0.0222 , Supplemental Figure $1 C$ ) fortify the notion that CEACAM1 dictates ASK1/p-p38 hepatic expression under cold-storage stress.

HMGB1, an evolutionarily conserved nuclear protein loosely bound to chromatin, is involved in DNA organization and regulation of transcription. When released by damaged cells, extracellular HMGB1 serves as an indicator of cell injury as well as a danger signal stimulating downstream inflammatory reactions via its interaction with sentinel TLR4 and upregulation of NF- $\kappa \mathrm{B}$, which leads to increased production/release of cytokines $(29,30)$. In the present study, cold stimulation increased liver/native hepatocyte ROS (4HNE) expression (Figure 2C and Figure 4C) and HMGB1 translocation (Figure 2, B and C, and Figure 4C). ROS production is critically involved in cytoplasmic translocation of HMGB1 in hepatocytes (31), whereas, conversely, hepatocyte-specific HMGB1 depletion enhances ROS production in the liver (32). This implies that sustained ROS generation and HMGB1 translocation and release may act synergistically to aggravate liver parenchymal cell loss under sustained cold stress. In our in vivo and in vitro experimental models, cold-stressed hepatocytes were TUNEL negative (nonapoptotic/necrotic, Figure 2C and unpublished observations), with increased ASK1/p-p38 signaling (Figure 3C and Figure 4A) and enhanced HMGB1 translocation (Figure 2C and Figure 4C) profiles. Hattori et al. recently found that ASK1/p-p38 signaling is essential in cold-stimulated cell death in a cell type-specific manner. They also documented the functional role of ferroptosis, an iron-dependent and oxidative programmed cell death leading to extensive DAMPs release (33), evidenced by cytoprotective effects of ferroptosis inhibitor (ferrostatin-1) in certain cell types under sustained cold stress (11). Despite an increasing number of studies focusing on ferroptosis in the mechanism of ischemic tissue injury in kidney, heart, and intestine (33-35), its contribution in the liver remains to be elucidated. By demonstrating distinctive ASK1/p-p38 versus cleaved caspase-3 expression patterns in mouse livers under ex vivo cold stress (18 hours) as compared with the posttransplant (3 hours) reperfusion phase (Figure 3, B and C), our results give a 
A ROC curve to determine optimal cutoff of CEACAM $1 / \beta$-actin for predicting EAD

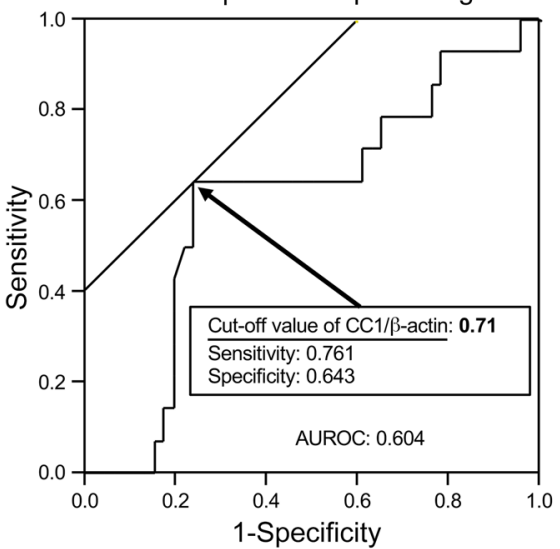

B

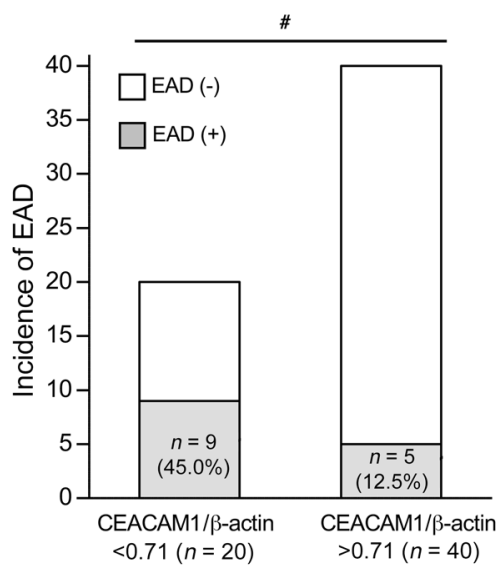

D

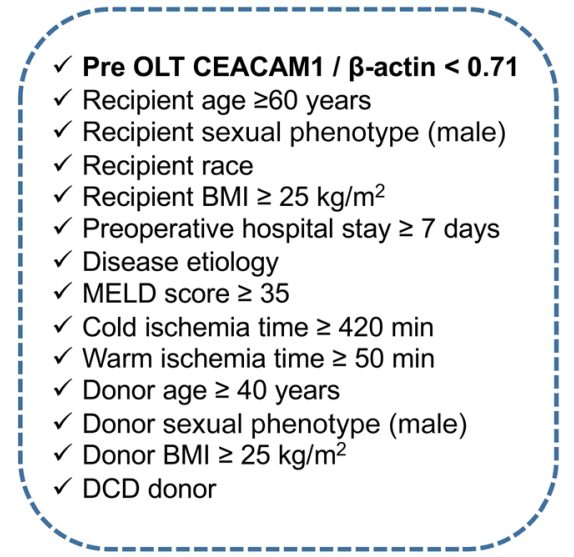

C

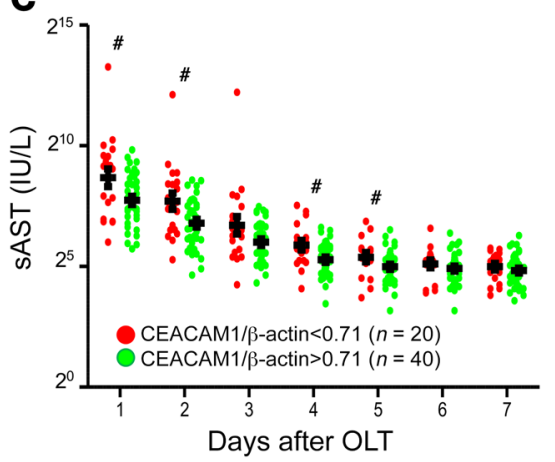

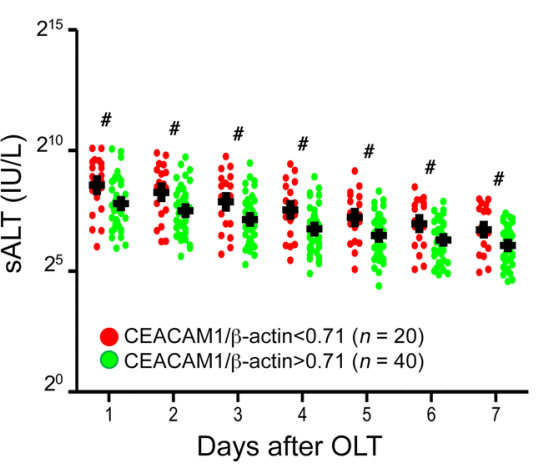

Multivariate analysis of $\mathrm{EAD}$

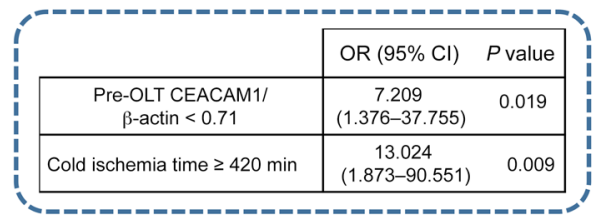

Figure 9. Pretransplant CEACAM1 expression dictates the incidence of EAD in human OLT patients. Pretransplant (after cold storage) human liver BX samples $(n=60)$ were analyzed by Western blots with $\beta$-actin normalization for CEACAM1 levels. (A) ROC analysis of CEACAM1/ $\beta$-actin for predicting EAD. Based on a ROC curve and Youden index on the basis of best accuracy in relation to EAD incidence, the CEACAM1/ $\beta$-actin cutoff value of 0.71 was determined. AUROC, area under the receiver operating characteristic curve. (B) Based on the optimal cutoff value (0.71), 60 human OLTs were classified into CEACAM1/ $\beta$-actin less than $0.71(n=20)$ and CEACAM1/ $\beta$-actin greater than 0.71 cases $(n=40)$, and the incidence of EAD was evaluated. ${ }^{\#} P<0.05$ (Fisher's exact test). (C) Serum AST and ALT levels at POD1-7. Data are mean \pm SEM. ${ }^{~} P<0.05$ (Mann-Whitney $U$ test). (D) Stepwise multivariate logistic regression analysis was performed to identify independent risk factors of EAD.

foothold for future investigations of putative inflammatory cell death mechanisms in murine and human IRI-OLT settings, e.g., cold storage-induced CC1/ASK1/p-p38/ROS-related cell death (ferroptosis?) versus warm reperfusion-induced TUNEL-positive necrosis/apoptosis. Indeed, in our ongoing study, incubation of donor mouse livers with ferroptosis inhibitor during cold storage alleviated the classic IRI phenotype in a WT $\rightarrow$ WT OLT model (unpublished observations).

In the pathophysiology of liver IRI, innate immune-driven activation in the reperfusion phase triggers hepatocellular damage, manifested by elevated SAST/sALT levels and increased frequency of TUNEL-positive (necrosis/apoptosis) cells. Meanwhile, ischemia itself causes initial hepatic damage and the release of DAMPs, such as HMGB1 and histone $\mathrm{H} 3$, which then ignite innate immune cascade in the early reperfusion phase. Interestingly, despite cold storage-triggered tissue damage with ample HMGB1/ histone H3 (Figure 2B), the hepatocellular injury in our study was not accompanied by increased frequency of TUNEL-positive cells (Figure 2C), indicating that a mechanism other than apoptosis/ necrosis cell death was at work. Our data stress the importance of CEACAM1 signaling to alleviate the aforementioned graft damage during cold stress (Figure 2), which in turn mitigated the postreperfusion inflammatory phenotype (Figure 1). However, as to whether and how hepatic CEACAM1 may directly influence hepatocyte apoptosis/necrosis remains to be elucidated. On the other hand, we have reported that Kupffer cells (liver-resident macrophages) undergo IR-triggered necroptosis (36). Although neither cold stress nor reperfusion significantly increased hepatic expression of RIP3 (a necroptosis marker) in the present study (Figure 3B), macrophage-specific RIP3 seems to be specifically enhanced during the reperfusion phase as compared with the cold-stress phase (unpublished observations). Hence, distinct cell death mechanisms/signaling pathways, likely dependent on cell type (hepatocyte, macrophage, etc.), timing (ischemia or reperfusion), and/or ischemia type (warm or cold), contribute to liver IRI-OLT pathogenesis. Since macrophages do express CEACAM1 (unpublished observations), whether and how macrophagespecific CEACAM1 can regulate necroptosis in IR-stressed livers awaits further study.

To estimate cold preservation-induced liver graft damage, we measured HMGB1 concentration in the hepatic effluent. The initial flush from cold-stored mouse livers (Figure 2B and Figure 
$5 A$ ) is unlikely to directly circulate in recipient blood in the clinical scenario because mouse liver grafts were perfused (lactate ringer solution) before revascularization, whereas human livers are routinely flushed before completion of the vascular reperfusion. As experimental and clinical surgery procedures refrain from extensive graft rinse to prevent tissue cell damage, especially sinusoidal endothelial stress (shear stress), extranuclear HMGB1 remaining despite flushing was possibly emitted by vascular reperfusion into host circulation to exacerbate recipient immune responses. In this context, aggravated OLT damage in CC1-deficient livers was accompanied by increased serum HMGB1 levels (Figure 1F) and enhanced innate immune activation after reperfusion (Figure 1, $\mathrm{D}$ and $\mathrm{F}-\mathrm{H})$. Likewise, low CEACAM1 expression in cold-stored human donor livers was associated with an increased postreperfusion innate-immune gene activation profile, including TLR4 (the canonical LPS and one of HMGB1 receptors), CD80, CD86, CXCL10, CD68, and Cathepsin G (Figure 8). Despite a variety of flushing clinical protocols, there is uncertainty as to the benefit of graft rinse or which protocol may be the most advantageous for transplantation outcomes $(37,38)$. Obviously, in addition to strategies alleviating graft injury under cold preservation stress, future studies are desired, to seek optimal hepatic rinsing protocols to suppress graft-derived DAMPs release, reduce innate-adaptive immune interface responses, and hence improve OLT outcomes.

CEACAM1 was recently discovered as a cellular ligand for TIM-3 ( $\mathrm{T}$ cell immunoglobulin domain and mucin domain-3) coexpressed with TIM- 3 on activated CD $4^{+}$T cells (39), whereas 2 other known TIM-3 ligands, Galectin-9 and HMGB1, are cellreleased soluble proteins (alarmins). Of note, our own studies have revealed that TIM-3-negative signaling dictates the severity of hepatic injury in a mouse warm IRI model (40) and may confer resistance against cold preservation damage in OLT $(41,42)$. As a proficient CEACAM1-TIM-3 signaling pathway is required to mediate $\mathrm{T}$ cell exhaustion in autoimmunity and antitumor immunity (39), we analyzed whether CEACAM1-TIM-3 crosstalk on host circulating $\mathrm{CD} 4^{+} \mathrm{T}$ cells might promote an exhaustion-type phenotype in IR-stressed livers and whether under dominant CEACAM1 signaling TIM $-3^{+} \mathrm{CD} 4^{+}-$exhausted $\mathrm{T}$ cells may inhibit the development/progression of IRI in OLT.

Our current translational study highlights the previously unappreciated cytoprotective function of CEACAM1 expression during the liver graft cold preservation phase, and identifies the hepatocyte ASK1/p-p38 signaling axis as the molecular homeostatic mechanism. This, what we believe to be novel finding, may have important practical ramifications, especially in the context of recently reported antifibrotic efficacy for a first-in-class ASK1 inhibitor (selonsertib) in a clinical trial in patients with NASH (28), the next global epidemic, which will require liver transplantation. Our results provide the rationale for preconditioning of IRI-prone low CEACAM1-expressing human liver grafts with ASK1 inhibitor to improve outcomes. In addition, by identifying the decreased CEACAM1 phenotype as a poor prognostic biomarker, one may envision that the CEACAM signal is enhanced with its exogenous homophilic ligand (CEACAM1-Fc fusion protein) (43) during normothermic perfusion aimed to "rejuvenate" suboptimal donor livers before implantation.

In conclusion, as a checkpoint regulator of IR stress and sterile inflammation, CEACAM1 may be considered as a denominator of hepatic tissue quality. If translated to clinical practice, and confirmed for other organs, our findings would have a major impact on transplant outcomes, as currently there is no reliable way to preoperatively assess donor organ quality.

\section{Methods}

Clinical liver transplant study. The study was approved by the UCLA Institutional Review Board (IRB protocol 13-000143, 18-000216). We performed a retrospective analysis of 60 adult patients (age $\geq 18$ years) who underwent OLT (May 2013-August 2015). All the recipients received routine standard of care and immunosuppressive therapy, as specified by UCLA liver transplant protocols. Recipients who underwent retransplant were excluded from the study. Donor livers, procured from donation after brain death or cardiac death with standardized techniques, were perfused with and stored in UW solution (Niaspan; Bristol-Meyers Squibb Pharma). Protocol Tru-Cut needle Bx were obtained from the left liver lobe and snap-frozen. Pretransplant Bx were obtained after liver cold storage at back table (before implantation) from 60 cases, and then Bx samples were analyzed by Western blots with $\beta$-actin normalization for CC1, ASK1, and p-p38. Posttransplant $\mathrm{Bx}$ were collected 2 hours after portal reperfusion (before the abdominal closure) from a corresponding 44 cases. MELD was used to assess the severity of liver disease. Cold ischemia time was defined as the time from the perfusion of the donor liver with UW solution to its removal from the cold storage for implantation. Warm ischemia time was defined as the time from removal from cold storage to establishment of liver graft reperfusion. Recipient blood was collected before and after OLT, and liver function was evaluated by sALT and SAST. EAD was defined by the presence of one or more of the following: bilirubin level of $\geq 10 \mathrm{mg} / \mathrm{dL}$ on POD7, prothrombin timeInternational Normalized Ratio (PT-INR) $\geq 1.6$ on POD7, or AST/ALT level of $>2000 \mathrm{U} / \mathrm{L}$ within the first 7 days (44).

Animals. Wild-type mice (Jackson Laboratory) and mice with global $C C 1$ genetic ablation (CC1-KO/CC1 ${ }^{-1-}$; courtesy of M. Kujawski, Beckman Research Institute, City of Hope, CA; originally generated by N. Beauchemin, McGill University, Montreal, Canada) (45), both C57BL/6 background and 6 to 8 weeks of age, were used. Animals were housed in the UCLA animal facility under specific pathogen-free conditions, received humane care according to the criteria outlined in the Guide for the Care and Use of Laboratory Animals (National Academies Press, 2011), and their use was reviewed and approved by UCLA Animal Research Committee.

Mouse orthotopic liver transplantation. We used a mouse model of ex vivo hepatic cold storage followed by orthotopic liver transplantation (OLT), as described by our group (46). To mimic the marginal human OLT setting, and to focus on putative hepatic CEACAM1 cytoprotective functions while avoiding confounding host allo-immune MHC responses, donor livers (WT or $\mathrm{CC1}^{-1 /}$; BL6) stored in UW solution $\left(4^{\circ} \mathrm{C} / 18\right.$ hours) were transplanted to syngeneic rather than allogeneic recipient mice. In separate experiments, donor livers were incubated with ASK1-specific small molecule inhibitor $(10 \mu \mathrm{g} / 15 \mathrm{~mL}$, MSC2032964A, R\&D Systems) throughout 18 hours of cold storage. Liver graft and serum samples were collected at 6 hours after reperfusion, the peak of hepatocellular damage in this model. Separate OLT recipient groups were monitored for survival. The sham group underwent the same procedures except for OLT. To study the influence of cold ischemia alone, some livers/liver flush (after infusion of $2 \mathrm{~mL}$ 
physiological saline via a cuff in the portal vein) were obtained shortly after completion of ex vivo storage.

Hepatocellular function assay. Serum AST/ALT levels were measured with Infinity AST/ALT Liquid Stable Reagent (Thermo Fisher Scientific) and validated with Validate GC3 (Maine Standards Company, LLC).

OLT histology and IRI grading. Formalin-fixed paraffin-embedded liver sections $(5 \mu \mathrm{m})$ were stained with H\&E. The severity of IRI was graded using Suzuki's criteria (47).

TUNEL assay. Cell death in liver sections $(5 \mu \mathrm{m})$ was detected by In Situ Apoptosis Detection Kit (Clontech) according to the manufacturer's protocol. Results were scored semiquantitatively by blindly counting the number of positive cells in $10 \mathrm{HPF} /$ section (48).

ELISA. Serum concentration of HMGB1 (MyBioSource) and MCP1 (Thermo Fisher Scientific) was measured by ELISA kit according to the manufacturer's protocol (49).

BMDM cultures. Femurs and tibias were removed from WT mice, and bone marrow-derived macrophages were generated as described (50). Cells were incubated (6 hours) with $100 \mu \mathrm{L}$ liver flush obtained from WT or CC1-KO cold-stored liver grafts.

Hepatocyte isolation and cultures. Primary mouse hepatocytes, isolated by a 2-stage collagenase perfusion method (51), were cultured with or without cold stress $\left(4^{\circ} \mathrm{C}\right)$ for the indicated time periods ( 2 hours/4 hours/6 hours). In some experiments, hepatocytes were transfected with ASK1-siRNA (Santa Cruz Biochemistry) using Lipofectamine reagent (Invitrogen) in advance of cold stimulation (6). Dead cells were detected using Live/Dead Cell Staining Kit II (PromoCell $\mathrm{GmbH}$ ) according to the manufacturer's protocol.

Western blot assay. Proteins were extracted from tissue and cell samples, and their concentration was measured using the BCA Protein Assay Kit (Thermo Fisher Scientific). An equal amount of protein was electrophoresed, blotted, incubated with primary Abs, secondary HRP-conjugated Abs, and developed. Primary Abs detecting HMGB1 (6893/D3E5), Histone H3 (4499/D1H2), cleaved caspase-3 (9664/5A1E), RIP3 (95702/D4G2A), CC1 (14771/D1P4T), ASK1 (8662/D11C9), p-p38 (4511/D3F9), Vinculin (VCL) (18799/E1E9V), and $\beta$-actin (12620/D6A8, Cell Signaling Technology) were used. To compare target protein expression in multiple human OLT samples, densitometry quantification was conducted as reported (50). Briefly, in a preliminary study, one of the Bx samples expressing all target proteins was chosen and assigned as a control sample. An equal amount of protein lysate from each sample was applied to each well or gel, and the target band intensity was expressed as relative band intensity to that of the positive control in the same gel. The target relative protein value was further normalized according to $\beta$-actin intensity.

Quantitative RT-PCR analysis. RNA extracted with the RNAse Mini Kit (Qiagen) was reverse-transcribed into cDNA. Quantitative PCR was performed using QuantStudio 3 (Applied Biosystems). The primer sequences are listed in Supplemental Table 6. The expression of the target gene was normalized to the housekeeping HPRT, $\beta 2 \mathrm{M}$, or GAPDH (52).

Immunofluorescence. Mouse liver samples were stained with rabbit anti-CD11b Ab (ab133357/EPR1344, Abcam) and rat anti-Ly6G Ab (551459/1A8, BD Biosciences). Hepatic CD11 b ${ }^{+} / \mathrm{Ly} 6 \mathrm{G}^{+}$cells were scored semiquantitatively by blindly counting cells in $10 \mathrm{HPF} /$ section $(\times 400)$. Mouse and human liver samples were stained with sheep anti-CC1 Ab (AF6480, R\&D Systems), rabbit anti-4HNE Ab (ab46545, Abcam), and rabbit anti-HMGB1 Ab (ab79823/EPR3507, Abcam). Mouse hepatocytes were stained using rabbit anti-4HNE Ab and rabbit anti-HMGB1 Ab. Signals were visualized with secondary Alexa Fluor Abs.

Statistics. For mouse experiments, comparisons between 2 groups or multiple groups were assessed using a Student's $t$ test or 1-way ANOVA followed by Tukey's honest significant difference (HSD) test, respectively. For human data, continuous values were analyzed by Mann-Whitney $U$ test and categorical variables by Fisher's exact test. A multivariable analysis based on a stepwise logistic regression model was used to identify independent predictive factors of EAD. Cumulative survival rates were estimated using the Kaplan-Meier method, and survival curves were compared using log-rank tests. Identification of an optimal $\mathrm{CM} 1 / \beta$-actin cutoff value was done via a receiver operating characteristics (ROC) analysis, and Youden's index as the basis for determining the best accuracy in relation to EAD incidence. All $P$ values were 2-tailed, and $P$ less than 0.05 was considered statistically significant. JMP for Windows 8.0 (SAS Institute) was used for statistical analyses.

Study approval. All human studies were approved by the UCLA Institutional Research Board (IRB protocol 13-000143) and written informed consent was received from participants before inclusion in the study. All mouse experiments were approved by the UCLA Animal Research Committee (ARC 1999-094).

\section{Author contributions}

$\mathrm{KN}, \mathrm{SK}, \mathrm{HH}, \mathrm{TI}$, and JWKW conceived and designed the study. $\mathrm{KN}, \mathrm{SK}, \mathrm{HH}, \mathrm{KK}$, and KJD acquired experimental data. SK, HH, and $\mathrm{HK}$ were responsible for surgical procedures. KN, TI, and FMK performed clinical data analyses. DWG, RAS, EFR, MK, FMK, and RWB were discussants and reviewed the manuscript. KN, SK, HH, TI, and JWKW drafted the manuscript. RWB and JWKW obtained funding. All authors read and edited the manuscript. The co-first authors are listed in reverse alphabetical order.

\section{Acknowledgments}

We thank Ko Takanashi (UCLA-Translational Pathology Core Laboratory) and Damla Oncel (UCLA) for immunohistochemical stain assistance, and Justine Aziz and Stephanie Younan for helping with clinical data collection. This work was supported by NIH grants P01 AI120944 (to JWKW and EFR), and R01 DK062357, DK107533, and DK102110 (to JWKW).

Address correspondence to: Jerzy W. Kupiec-Weglinski, Dumont-UCLA Transplant Center, 77-120 CHS, 10833 Le Conte Avenue, Los Angeles, California 90095, USA. Phone: 310.825.4196; Email: jkupiec@mednet.ucla.edu.

KN's present address is: Department of Surgery, Kyoto University, Kyoto, Japan, and Department of Surgery, Nishi-Kobe Medical Center, Hyogo, Japan.
1. Hudcova J, Scopa C, Rashid J, Waqas A, Ruthazer R, Schumann R. Effect of early allograft dysfunction on outcomes following liver transplantation. Clin Transplant. 2017;31(2):e12887.
2. Zhai Y, Petrowsky H, Hong JC, Busuttil RW, Kupiec-Weglinski JW. Ischaemia-reperfusion injury in liver transplantation--from bench to bedside. Nat Rev Gastroenterol Hepatol.
2013;10(2):79-89.

3. Gujral JS, Bucci TJ, Farhood A, Jaeschke H. Mechanism of cell death during warm hepatic ischemia-reperfusion in rats: apoptosis or necrosis? 
Hepatology. 2001;33(2):397-405.

4. Jaeschke H, Lemasters JJ. Apoptosis versus oncotic necrosis in hepatic ischemia/reperfusion injury. Gastroenterology. 2003;125(4):1246-1257.

5. Baskin-Bey ES, et al. Clinical trial of the pan-caspase inhibitor, IDN-6556, in human liver preservation injury. Am J Transplant. 2007;7(1):218-225.

6. Kageyama S, et al. Recombinant relaxin protects liver transplants from ischemia damage by hepatocyte glucocorticoid receptor: From benchto-bedside. Hepatology. 2018;68(1):258-273.

7. Yang F, et al. TNF $\alpha$-mediated necroptosis aggravates ischemia-reperfusion injury in the fatty liver by regulating the inflammatory response. Oxid Med Cell Longev. 2019;2019:2301903.

8. Karhumäki P, Tiitinen SL, Turpeinen H, Parkkinen J. Inhibition of ERK1/2 activation by phenolic antioxidants protects kidney tubular cells during cold storage. Transplantation. 2007;83(7):948-953.

9. Gon Y, Hashimoto S, Matsumoto K, Nakayama $\mathrm{T}$, Takeshita I, Horie T. Cooling and rewarming-induced IL-8 expression in human bronchial epithelial cells through p38 MAP kinase-dependent pathway. Biochem Biophys Res Commun. 1998;249(1):156-160.

10. Fujiwara Y, Denlinger DL.p38 MAPK is a likely component of the signal transduction pathway triggering rapid cold hardening in the flesh fly Sarcophaga crassipalpis. JExp Biol. 2007; 210(Pt 18):3295-3300.

11. Hattori K, Ishikawa H, Sakauchi C, Takayanagi S, Naguro I, Ichijo H. Cold stress-induced ferroptosis involves the ASK1-p38 pathway. EMBO Rep. 2017;18(11):2067-2078.

12. Rees-Jones RW, Taylor SI. An endogenous substrate for the insulin receptor-associated tyrosine kinase. J Biol Chem. 1985;260(7):4461-4467.

13. Ocklind C, Obrink B. Intercellular adhesion of rat hepatocytes. Identification of a cell surface glycoprotein involved in the initial adhesion process. J Biol Chem. 1982;257(12):6788-6795.

14. Poy MN, et al. CEACAM1 regulates insulin clearance in liver. Nat Genet. 2002;30(3):270-276.

15. Ghosh S, et al. Mice with null mutation of Ceacam I develop nonalcoholic steatohepatitis. Hepat Med.2010;2010(2):69-78.

16. Horst AK, Najjar SM, Wagener C, Tiegs G. CEACAM1 in liver injury, metabolic and immune regulation. Int J Mol Sci. 2018;19(10):E3110.

17. Nakamura K, Kageyama S, Kupiec-Weglinski JW. The evolving role of neutrophils in liver transplant ischemia-reperfusion injury. Curr Transplant Rep. 2019;6(1):78-89.

18. Awad EM, et al. Cold induces reactive oxygen species production and activation of the NF-kappa $B$ response in endothelial cells and inflammation in vivo. J Thromb Haemost. 2013;11(9):1716-1726.

19. Guo X, et al. Regulation of the severity of neuroinflammation and demyelination by TLR-ASK1-p38 pathway. EMBO Mol Med. 2010;2(12):504-515.

20. Horst AK, et al. Carcinoembryonic antigen-related cell adhesion molecule 1 controls IL-2-dependent regulatory T-cell induction in immune-mediated hepatitis in mice. Hepatology.
2018;68(1):200-214.

21. Dery KJ, Gaur S, Gencheva M, Yen Y, Shively JE, Gaur RK. Mechanistic control of carcinoembryonic antigen-related cell adhesion molecule-1 (CEACAM1) splice isoforms by the heterogeneous nuclear ribonuclear proteins hnRNP L, hnRNP A1, and hnRNP M. J Biol Chem. 2011;286(18):16039-16051.

22. Chen L, et al. The short isoform of the CEACAM1 receptor in intestinal $\mathrm{T}$ cells regulates mucosal immunity and homeostasis via Tfh cell induction. Immunity. 2012;37(5):930-946.

23. Ichijo $\mathrm{H}$, et al. Induction of apoptosis by ASK1, a mammalian MAPKKK that activates SAPK/ JNK and p38 signaling pathways. Science. 1997;275(5296):90-94.

24. Imoto K, et al. Impact of mitochondrial reactive oxygen species and apoptosis signal-regulating kinase 1 on insulin signaling. Diabetes. 2006;55(5):1197-1204.

25. Saito J, et al. Regulation of apoptosis signalregulating kinase 1 by protein phosphatase 2Cepsilon. Biochem J. 2007;405(3):591-596.

26. Tobiume K, Saitoh M, Ichijo H. Activation of apoptosis signal-regulating kinase 1 by the stress-induced activating phosphorylation of pre-formed oligomer. J Cell Physiol. 2002;191(1):95-104.

27. Challa TD, et al. Liver ASK1 protects from nonalcoholic fatty liver disease and fibrosis. EMBO Mol Med. 2019;11(10):e10124.

28. Loomba R, et al. The ASK1 inhibitor selonsertib in patients with nonalcoholic steatohepatitis: A randomized, phase 2 trial. Hepatology. 2018;67(2):549-559.

29. Nakamura K, et al. Soluble thrombomodulin attenuates sinusoidal obstruction syndrome in rat through suppression of high mobility group box 1. Liver Int. 2014;34(10):1473-1487.

30. Klune JR, Dhupar R, Cardinal J, Billiar TR, Tsung A. HMGB1: endogenous danger signaling. $\mathrm{Mol}$ Med. 2008;14(7-8):476-484.

31. Tsung A, et al. HMGB1 release induced by liver ischemia involves Toll-like receptor 4 dependent reactive oxygen species production and calcium-mediated signaling. J Exp Med. 2007;204(12):2913-2923.

32. Huang H, et al. Hepatocyte-specific highmobility group box 1 deletion worsens the injury in liver ischemia/reperfusion: a role for intracellular high-mobility group box 1 in cellular protection. Hepatology. 2014;59(5):1984-1997.

33. Li W, et al. Ferroptotic cell death and TLR4/ Trif signaling initiate neutrophil recruitment after heart transplantation. J Clin Invest. 2019;129(6):2293-2304.

34. Pefanis A, Ierino FL, Murphy JM, Cowan PJ. Regulated necrosis in kidney ischemia-reperfusion injury. Kidney Int. 2019;96(2):291-301.

35. Li Y, et al. Ischemia-induced ACSL4 activation contributes to ferroptosis-mediated tissue injury in intestinal ischemia/reperfusion. Cell Death Differ. 2019;26(11):2284-2299.

36. Yue S, Zhou H, Wang X, Busuttil RW, KupiecWeglinski JW, Zhai Y. Prolonged ischemia triggers necrotic depletion of tissue-resident macrophages to facilitate inflammatory immune activation in liver ischemia reperfusion injury. JImmunol. 2017;198(9):3588-3595.

37. Gurusamy KS, Naik P, Abu-Amara M, Fuller B, Davidson BR. Techniques of flushing and reperfusion for liver transplantation. Cochrane Database Syst Rev. 2012;(3):CD007512.

38. Houben P, et al. Graft rinse prior to reperfusion in liver transplantation: literature review and online survey within the Eurotransplant community. Transpl Int. 2015;28(11):1291-1298.

39. Huang YH, et al. CEACAM1 regulates TIM-3mediated tolerance and exhaustion. Nature. 2015;517(7534):386-390.

40. Uchida Y, et al. T-cell immunoglobulin mucin-3 determines severity of liver ischemia/reperfusion injury in mice in a TLR4-dependent manner. Gastroenterology. 2010;139(6):2195-2206.

41. Liu Y, et al. Recipient T cell TIM-3 and hepatocyte galectin-9 signalling protects mouse liver transplants against ischemia-reperfusion injury. J Hepatol. 2015;62(3):563-572.

42. Liu Y, et al. Negative CD4 + TIM-3 signaling confers resistance against cold preservation damage in mouse liver transplantation. Am J Transplant. 2015;15(4):954-964.

43. Iijima $H$, et al. Specific regulation of $T$ helper cell 1-mediated murine colitis by CEACAM1. J Exp Med. 2004;199(4):471-482.

44. Nakamura K, et al. Sirtuin 1 attenuates inflammation and hepatocellular damage in liver transplant ischemia/reperfusion: from mouse to human. Liver Transpl. 2017;23(10):1282-1293.

45. Blau DM, et al. Targeted disruption of the Ceacam1 (MHVR) gene leads to reduced susceptibility of mice to mouse hepatitis virus infection. J Virol. 2001;75(17):8173-8186.

46. Kageyama S, Nakamura K, Ke B, Busuttil RW, Kupiec-Weglinski JW. Serelaxin induces Notch1 signaling and alleviates hepatocellular damage in orthotopic liver transplantation. Am J Transplant. 2018;18(7):1755-1763.

47. Suzuki S, Toledo-Pereyra LH, Rodriguez FJ, Cejalvo D. Neutrophil infiltration as an important factor in liver ischemia and reperfusion injury. Modulating effects of FK506 and cyclosporine. Transplantation. 1993;55(6):1265-1272.

48. Nakamura K, et al. Heme oxygenase-1 regulates sirtuin-1-autophagy pathway in liver transplantation: from mouse to human. Am J Transplant. 2018;18(5):1110-1121.

49. Kageyama S, et al. Recipient HO-1 inducibility is essential for posttransplant hepatic HO-1 expression and graft protection: from bench-to-bedside. Am J Transplant. 2019;19(2):356-367.

50. Nakamura K, et al. Macrophage heme oxygenase1-SIRT1-p53 axis regulates sterile inflammation in liver ischemia-reperfusion injury. J Hepatol. 2017;67(6):1232-1242.

51. Nakamura K, et al. Antibiotic pretreatment alleviates liver transplant damage in mice and humans. J Clin Invest. 2019;129(8):3420-3434.

52. Kadono K, et al. Heme Oxygenase-1 dictates innate-adaptive immune phenotype in human liver transplantation. Arch Biochem Biophys. 2019;671:162-166. 